\title{
Phytochemical Investigation of New Algerian Lichen Species: Physcia Mediterranea Nimis
}

\author{
Marwa Kerboua ${ }^{1}$, Monia Ali Ahmed ${ }^{1}$, Nsevolo Samba ${ }^{2,3}{ }^{\mathbb{D}}$, Radhia Aitfella-Lahlou ${ }^{2,4,5}{ }^{\mathbb{D}}$, Lucia Silva ${ }^{2,4}$, \\ Juan F. Boyero ${ }^{6}$, Cesar Raposo ${ }^{6}$ and Jesus Miguel Lopez Rodilla ${ }^{2,4, * \text { (D) }}$
}

1 Laboratory of Vegetal Biology and Environment, Biology Department, Badji Mokhtar University, Annaba 23000, Algeria; kerbouamarwa@gmail.com (M.K.); serradj.monia@gmail.com (M.A.A.)

2 Chemistry Department, University of Beira Interior, 6201-001 Covilha, Portugal; nsevolo.samba@ubi.pt (N.S.); ra.aitfella@ubi.pt (R.A.-L.); mlas@ubi.pt (L.S.)

3 Department of Clinical Analysis and Public Health, University Kimpa Vita, Uige 77, Angola

4 Fiber Materials and Environmental Technologies (FibEnTech), University of Beira Interior, 6201-001 Covilhã, Portugal

5 Laboratory of Valorisation and Conservation of Biological Resources, Biology Department, Faculty of Sciences, University M'Hamed Bougara, Boumerdes 35000, Algeria

6 Department of Analytical Chemistry, Nutrition and Food Science, Faculty of Chemistry, Chromatographic and mass analysis service (NUCLEUS), University of Salamanca, 37008 Salamanca, Spain; jfbb@usal.es (J.F.B.); raposo@usal.es (C.R.)

* Correspondence: rodilla@ubi.pt; Tel.: +351-275-241-306

Citation: Kerboua, M.; Ahmed, M.A.; Samba, N.; Aitfella-Lahlou, R.; Silva, L.; Boyero, J.F.; Raposo, C.; Lopez Rodilla, J.M. Phytochemical Investigation of New Algerian Lichen Species: Physcia Mediterranea Nimis. Molecules 2021, 26, 1121. https:// doi.org/10.3390/molecules26041121

Academic Editor: Maria Atanassova

Received: 21 January 2021

Accepted: 18 February 2021

Published: 20 February 2021

Publisher's Note: MDPI stays neutral with regard to jurisdictional claims in published maps and institutional affiliations.

Copyright: (C) 2021 by the authors. Licensee MDPI, Basel, Switzerland. This article is an open access article distributed under the terms and conditions of the Creative Commons Attribution (CC BY) license (https:/ / creativecommons.org/licenses/by/ $4.0 /)$.

\begin{abstract}
The present study provides new data concerning the chemical characterisation of Physcia mediterranea Nimis, a rare Mediterranean species belonging to the family Physciaceae. The phytochemical screening was carried out using GC-MS, HPLC-ESI-MS-MS, and NMR techniques. Hot extraction of $n$-hexane was carried out, followed by separation of the part insoluble in methanol: wax (WA-hex), from the part soluble in methanol (ME-hex). GC-MS analysis of the ME-hex part revealed the presence of methylbenzoic acids such as sparassol and atraric acid and a diterpene with a kaurene skeleton which has never been detected before in lichen species. Out of all the compounds identified by HPLC-ESI-MS-MS, sixteen compounds are common between WA-hex and ME-hex. Most are aliphatic fatty acids, phenolic compounds and depsides. The wax part is characterised by the presence of atranorin, a depside of high biological value. Proton $1 \mathrm{H}$ and carbon 13C NMR have confirmed its identification. Atranol, chloroatranol (depsides compound), Ffukinanolide (sesquiterpene lactones), leprolomin (diphenyl ether), muronic acid (triterpenes), and ursolic acid (triterpenes) have also been identified in ME-hex. The results suggested that Physcia mediterranea Nimis is a valuable source of bioactive compounds that could be useful for several applications as functional foods, cosmetics, and pharmaceuticals.
\end{abstract}

Keywords: Algerian lichen; Physcia mediterranea Nimis; bioactive compounds; n-hexane extract; wax; GC-MS; HPLC-ESI-MS-MS; NMR

\section{Introduction}

From the Greek word, "leikhen" lichen was first used to designate a plant in the 4th century BC by Theophraste [1]. This small organism has been integrated into the fungal kingdom and results from the symbiotic association of a fungus called mycobiont and a green alga or a cyanobacterium called photobiont [2,3]. An estimated more than 17,000 species of lichen exist today, extending from the tropics to the polar regions [4]. The symbiosis gives lichens a specific structure and reproduction to each constituent alone. Unlike higher plants, they have no root, stem, or leaf, but a rudimentary vegetative apparatus: the thallus [5]. Moreover, they grow on a wide variety of substrates including soil, bark, bare rock surfaces, leaves of vascular plants, barnacle shells, and other lichens [4]. 
The mycobiont plays the most determining role in the morphology and structure of the lichen [6]. The thallus carries the elements necessary for reproduction. A great diversity of shapes and colours defines seven main types of lichens [7]. These organisms tolerate large temperature variations, extreme desiccation, and intense exposure to ultraviolet [8]. In addition, this tolerance to light and desiccation is better in lichen compared to isolated partners [9]. As a two or even multiple partnerships, lichens can respond more sensitive to different environmental signals in complex ecosystems than algae and fungi growing unprotected and living aposymbiotically according to Reyes et al., 1996 [10].

Lichens produce many unusual secondary metabolites that have not been discovered in other plants [11]. The uniqueness of many lichen substances attracted the first chemists' attention in the mid-19th century. Before this, they were used as dyes for textiles and as additives for soap and perfume making, and a considerable number for healing diseases [12,13]. Lichens produce more than 800 potentially bioactive compounds [14-16]. Among these compounds are nitrogen, phosphorus and sulfur, polyols, carbohydrates, aliphatic and cycloaliphatic compounds, aromatic compounds, meta- and para-depsides, and depsidones, dibenzofurans, diphenylethers, naphtopyrans, biphenyls, diphenylmethanes, nostoclides, xanthones, quinones, naphthoquinones, and usnic acid. Esters, terpenes, steroids, terphenylquinones, and pulvinic acid derivatives also occur [16-18]. The majority of lichenic substances are small aromatic polyketides. They are synthesised during the mutualistic relationship (symbiosis) of lichens with green photobionts $[13,19]$. These substances are often absent in cyanobacterial lichens replaced by other interesting secondary metabolites, e.g., terpenes and terpenoids $[20,21]$.

Recently, lichens have attracted several researchers worldwide to review their therapeutic and cosmetic value in traditional medicine. A wide range of species have revealed effective biological activities such as antioxidant, cardiovascular protectors, antimicrobial, anti-inflammatory, analgesic, antipyretic, antiviral, anti-insecticidal, antibiotic, antifungal, antidiabetic, and anticancer [22-31]. Various applications of lichen extracts as a treatment for skin conditions, wounds, respiratory and digestive problems, as well as for obstetric and gynaecological problems have also been recorded [32]. The pharmacological potential of lichens is due to the presence of unique secondary metabolites which subsequently became excessively attractive to the pharmaceutical and cosmetic industries. As a result, a multitude of optimised processes for the quantitative extraction of these lichen metabolites has been developed [33].

In Algeria, several investigations have been carried out to explore their diversity [34-45]. More than 1085 species of lichens have been identified, 64 of which are endemic [41]. Most research has been focused on their use as bioindicators of air pollution [42,43,46-49]. However, many regions remain unexplored, and research reports on lichen chemistry in this richly diverse country are very limited.

In the present investigation, we analysed the phytochemical profile of Physcia mediterranea Nimis (Figure 1), belonging to the family of Physicaceae. The lichen, a new species recently identified in Algeria, was collected in the El-Kala National Park, a remarkable and culturally rich region with more than 117 species of lichens identified. Several species of this area, both plant and animal, are protected in Algeria and belong to the IUCN (International Union for Conservation of Nature) red list. Indeed, the National park of El-Kala was classified as "Reserve of the biosphere", by UNESCO, on 17 December 1990 [50]. It can be concluded that the Algerian species of lichens that have been little or not studied deserve special attention with research involving both the chemical part and the biological one. This would allow us to increase the contribution to discovering new compounds that may serve as models for new drugs with therapeutic properties. The literature search reveals that still no studies have been done on Physcia mediterranea Nimis species' products. Therefore, our work aims to isolate and investigate the chemical constituents in different organic lichen extracts from Physcia mediterranea Nimis using GC-MS, followed by HPLC-ESI-MS-MS and NMR analysis. 


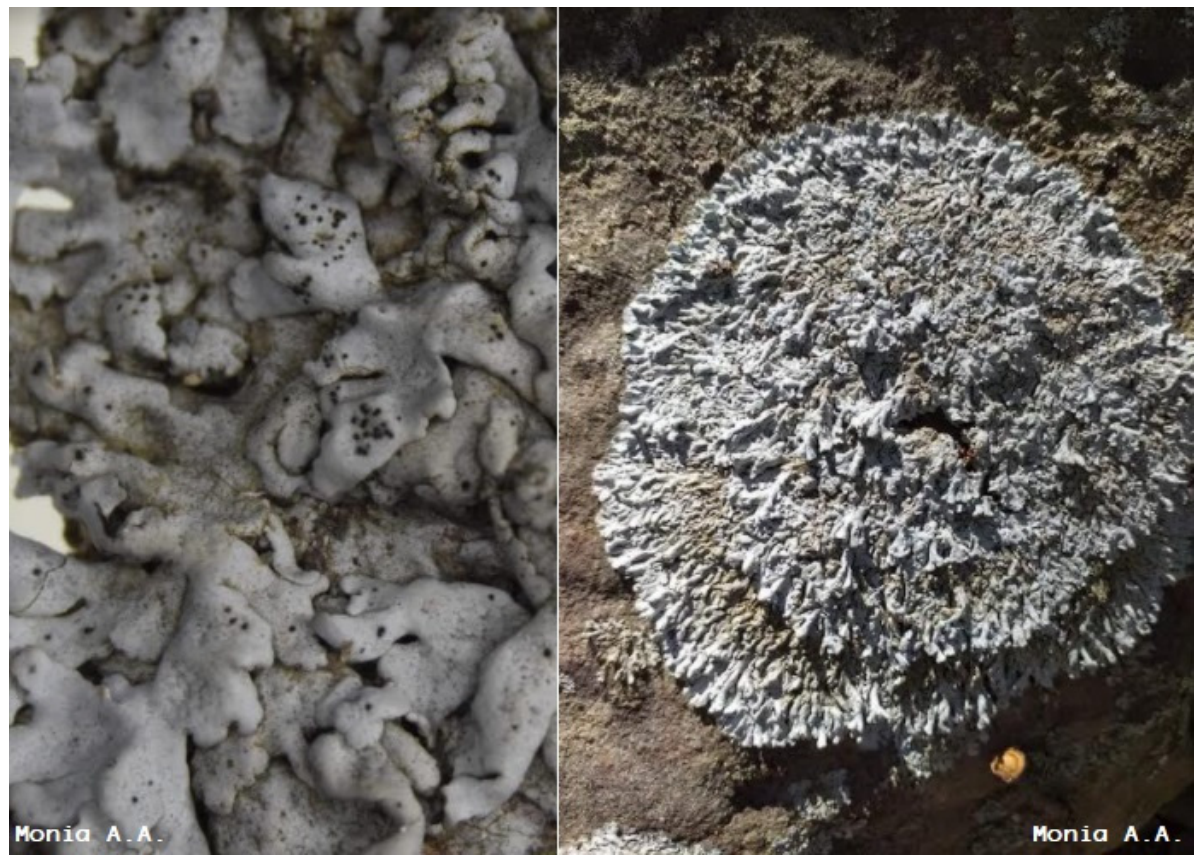

Figure 1. Algerian Physcia mediterranea Nimis photographed in the national park of El Kala, Algeria.

\section{Results and Discussion}

\subsection{GC-MS Analysis}

The GC-MS analysis of ME-hex extract is shown in Figure 2. The analysis allowed us to detect ten components. Nine products were identified by comparing their mass spectra with reference spectra from the NIST and Wiley databases (Table 1). The major chemical compounds identified in ME-hex from Physcia mediterranea included derivatives of methylbenzoic acids (56\%) and free fatty acids (36.1\%). A diterpene with a Kauran skeleton ((-)-ent-Kauran-16a-ol) (Figure 3) was also identified (3.8\%) [18]. Only 2.2\% of the entire extract was not identified.

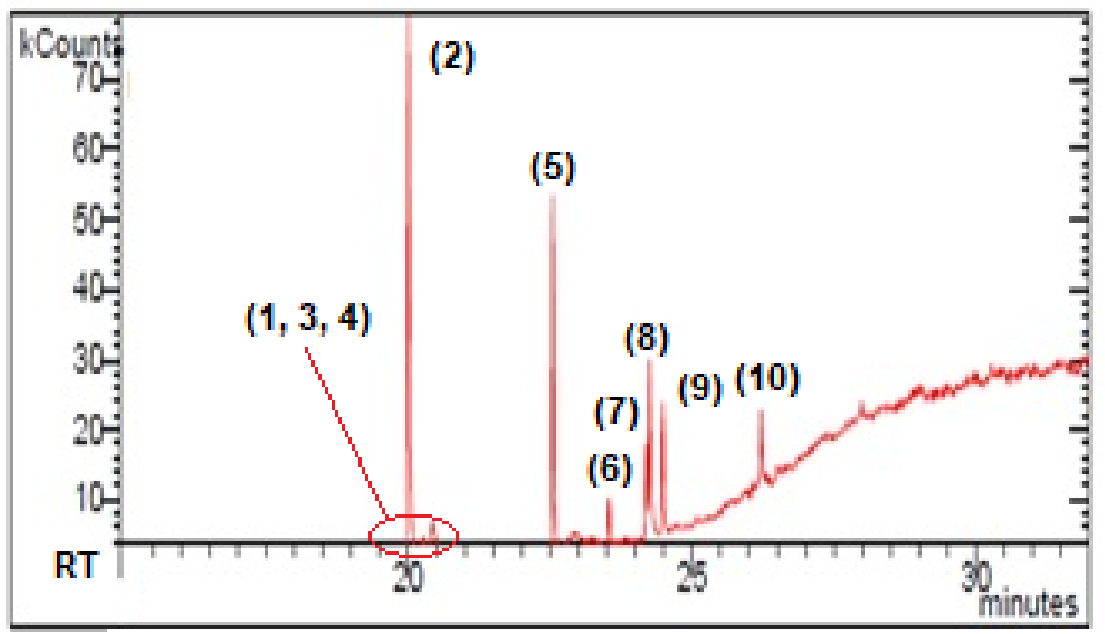

Figure 2. GC-MS chromatogram of Physcia mediterranea (ME-hex part). RT = retention time. (1), (2), (3), $(4)$ = derivatives of methylbenzoic acids; (5), (6), (7), (8) = fatty acids; (9) = diterpene; (10) = unknown. 
Table 1. Identification of metabolites in ME-hex of Physcia mediterranea by GC-MS analysis.

\begin{tabular}{|c|c|c|c|c|c|c|}
\hline $\mathbf{N}^{\circ}$ & RT & Compound & Mass & $\%$ & Synonyms & \\
\hline 1 & $19: 56$ & $\begin{array}{c}\text { Methyl } \\
\text { 2-hydroxy-4-methoxy- } \\
\text { 6-methylbenzoate }\end{array}$ & 196 & 0.3 & $\begin{array}{l}\text { Orsellinic Acid Methyl } \\
\text { Ester 4-Methyl Ether } \\
\text { (Sparassol) }\end{array}$ & $\mathrm{C}_{10} \mathrm{H}_{12} \mathrm{O}_{4}$ \\
\hline 2 & 20:01 & $\begin{array}{c}\text { Methyl } \\
\text { 2,4-dihydroxy-3,5,6- } \\
\text { trimethylbenzoate }\end{array}$ & 210 & 52.3 & $\begin{array}{l}\text { Derivatives of } \\
\text { methylbenzoic acids }\end{array}$ & $\mathrm{C}_{11} \mathrm{H}_{14} \mathrm{O}_{4}$ \\
\hline 3 & $20: 43$ & $\begin{array}{l}\text { Methyl } \\
\text { 4-hydroxy-2-methoxy- } \\
\text { 3,6-dimethylbenzoate }\end{array}$ & 210 & 1.1 & 2-metoxy-Atraric acid & $\mathrm{C}_{10} \mathrm{H}_{12} \mathrm{O}_{4}$ \\
\hline 4 & $20: 64$ & $\begin{array}{l}\text { Methyl 2,4-dihydroxy- } \\
\text { 3,6-dimethylbenzoate }\end{array}$ & 196 & 2.3 & Atraric acid & $\mathrm{C}_{10} \mathrm{H}_{12} \mathrm{O}_{4}$ \\
\hline 5 & 22:55 & Methyl hexadecanoate & 270 & 24.2 & Palmitic acid & $\mathrm{C}_{16} \mathrm{H}_{32} \mathrm{O}_{2}$ \\
\hline 6 & $23: 53$ & $\begin{array}{l}\text { 13-Methyl-17-norkaur- } \\
\text { 15-ene }\end{array}$ & 272 & 2.4 & Linoleic acid & $\mathrm{C}_{18} \mathrm{H}_{32} \mathrm{O}_{2}$ \\
\hline 7 & $24: 19$ & $\begin{array}{l}\text { Methyl cis, cis-9,12- } \\
\text { octadecadienoate }\end{array}$ & 294 & 3.2 & Oleic acid & $\mathrm{C}_{18} \mathrm{H}_{34} \mathrm{O}_{2}$ \\
\hline 8 & $24: 25$ & $\begin{array}{c}\text { Methyl } \\
\text { (Z)-9-octadecenoate }\end{array}$ & 296 & 4.9 & Stearic acid & $\mathrm{C}_{18} \mathrm{H}_{34} \mathrm{O}_{2}$ \\
\hline 9 & $24: 48$ & Methyloctadecanoate & 298 & 3.8 & $(-)$-ent-Kauran-16 $\alpha$-ol & $\mathrm{C}_{2} \mathrm{O}_{34} \mathrm{O}$ \\
\hline 10 & $26: 21$ & Unknown & 376 & 2.2 & Unknown & \\
\hline $\mathrm{T} \%$ & & & & 95.5 & & \\
\hline
\end{tabular}

$\mathrm{RT}=$ retention time; $\mathrm{T} \%$ = total of compounds identified (\%) in the extracts.

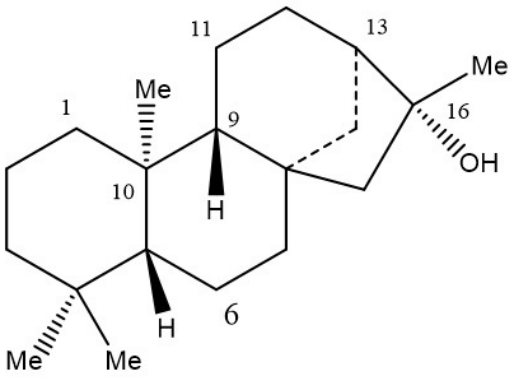

(a)

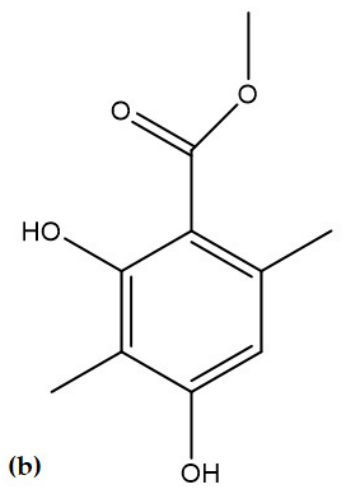

(b)

Figure 3. Chemical structure of (a) (-)-ent-Kauran-16a-ol and (b) Atraric acid.

Derivatives of methylbenzoic acids are commonly present in different genera of lichen-like Stereocaulon halei [51], Parmotrema mesotropum [52], Cassipourea malosana [53], Cetraria islandica [54], Usnea longissima [55,56], Stereocaulon paschale [57], and Parmelia sulcata Taylor [58]. Atraric acid (Figure 2), widely present in some species such as Hypogymnia physodes, Evernia prunastri, and Parmelia sulcata, growing on the same host tree (Prunus domestica) [59], can be in the free or complexed form and serves as a basis for the composition of depsides and depsidones [60]. It is considered a specific antagonist of androgen receptors and therefore inhibits human prostate cancer growth [60,61]. Atraric acid shows nematocidal, antioxidant, antimicrobial, and anti-inflammatory properties in vitro, and inhibits carrageenan-induced oedema and wound healing activity in vivo [62,63].

Lichens contain many of the fatty acids commonly found in higher plants [64]. Indeed, many common lichen genera include species with multiple strains of fatty acids [65-67]. These lipid profiles, most often treated as chemotypes, have been used by many researchers 
to taxonomically classify certain lichens, such as Cladonia [68], Lepraria [69], Parmelia [70], Tephromela s.lat [71], and Mycoblastus sanguinarius [72]. Although they are widely present in some genera, fatty acids were of no taxonomic importance and were omitted during the first chemical studies on lichens [72-74]. By chemical and biochemical comparisons, a mechanistic relationship between polyketide and fatty acid biosynthesis has been recognised. The carbon backbones of the molecules are assembled by successive condensation of acyl units [75]. Another important fatty acid role was cell signal transduction [76] as well as chemical protection. Therefore, they allow the lichens to survive as environmental conditions change [8-10].

In our study, palmitic acid is present in high concentration (24\%) compared to oleic $(4.2 \%)$, stearic acid (3.8\%), and linoleic (3.2\%) acid (Table 1 and Figure 2$)$. Various works using different growing conditions explain the variations in fatty acid in lichens $[77,78]$. Molina et al. (2003) studied the lichen Physconia distorta and suggested a close relationship between the synthesis of secondary metabolites and fatty acid metabolism. Mycobiota grown in a glucose-enriched medium favoured the production of fatty acids [79]. Another important factor that could influence the production of fatty acids in lichens is temperature. According to several studies, the degree of unsaturation varies with the season and decreases with increasing temperature $[80,81]$. In the thallus of Teloschistes flavicans, the saturated fatty acids, palmitic and stearic, were more abundant in February. In contrast, in August, when the average temperature was $23^{\circ} \mathrm{C}$, there was an increase in oleic and linoleic fatty acids [82].

In addition to the stress due to the decrease in temperature, nitrogen deprivation and light intensity are also known to promote fatty acid accumulation [80,82,83]. However, these factors are not necessarily the unique parameters that determine the fatty acid content, but rather genetics, combined with environmental conditions (e.g., altitude, air pollution, seasonal effects), must also be taken into account $[67,84]$.

\subsection{HPLC-ESI-MS-MS Analysis}

\subsubsection{WA-hex Extract Analysis of Physcia mediterranea}

Forty-five peaks (Figure S1) were detected for the first time in a WA-hex (Table 2) using LC/ESI/MS/MS in negative mode. Identifying major compounds was simplified by interpreting their MS/MS spectra, provided in our system resource and comparison with the literature. The 37 compounds identified were mainly paraconic and aliphatic acids, depsides (aromatic polyketides), phenolic compounds, and diterpenes. Only eight compounds could not be identified. The representative chemical structures are presented in Figure S2.

Table 2. Identification of metabolites in WA-hex of Physcia mediterranea by UHPLC/ESI/MS/MS.

\begin{tabular}{ccccccc}
\hline $\mathbf{N}^{\circ}$ & $\mathbf{R T}$ & {$[\mathbf{M}-\mathbf{H}]^{-}$} & $\mathbf{T M}$ & $\mathbf{M M}$ & $\mathbf{M T}$ & Compounds Identification \\
\hline 1 & 0.03 & 146.9397 & 147.9475 & $\mathrm{C}_{4} \mathrm{H}_{4} \mathrm{O}_{6}$ & $\mathrm{~A}$ & Dihydroxyfumaric acid \\
\hline 2 & 1.80 & 116.9276 & 117.9354 & $\mathrm{C}_{4} \mathrm{H}_{6} \mathrm{O}_{4}$ & $\mathrm{~A}$ & Butendioic acid \\
\hline 3 & 2.72 & 187.0971 & 188.1049 & $\mathrm{C}_{9} \mathrm{H}_{16} \mathrm{O}_{2}$ & $\mathrm{~A}$ & 3,5-dimethoxyciclohexanecarboxylic acid \\
\hline 4 & 5.51 & 163.0395 & 164.0473 & $\mathrm{C}_{9} \mathrm{H}_{8} \mathrm{O}_{3}$ & B & p-Coumaric acid \\
\hline 5 & 6.57 & 227.1286 & 228.1364 & $\mathrm{C}_{12} \mathrm{H}_{20} \mathrm{O}_{4}$ & $\mathrm{~A}$ & Trans-dodec-2-enedioic acid (traumatic acid) \\
\hline 6 & 7.46 & 282.2077 & 283.2155 & $\mathrm{C}_{16} \mathrm{H}_{29} \mathrm{NO}_{3}$ & $\mathrm{~A}$ & N-dodecanoyl-L-Homoserine lactone \\
\hline 7 & 8.09 & 174.9556 & 175.9634 & $\mathrm{C}_{5} \mathrm{H}_{4} \mathrm{O}_{7}$ & $\mathrm{~A}$ & 2-Hydroxy-3,4-dioxopentanedioc acid \\
\hline 8 & 8.15 & 293.1762 & 294.1840 & $\mathrm{C}_{17} \mathrm{H}_{26} \mathrm{O}_{4}$ & $\mathrm{~B}$ & Gingerol \\
\hline 10 & 14.37 & 295.2280 & 296.2358 & $\mathrm{C}_{18} \mathrm{H}_{32} \mathrm{O}_{3}$ & $\mathrm{~A}$ & 18-Hydroxylinoleic acid \\
\hline 11 & 15.36 & 177.0186 & 178.0264 & $\mathrm{C}_{9} \mathrm{H}_{6} \mathrm{O}_{4}$ & $\mathrm{~B}$ & 6,7-Dihydroxycoumarin (esculetin) \\
\hline
\end{tabular}


Table 2. Cont.

\begin{tabular}{|c|c|c|c|c|c|c|}
\hline $\mathbf{N}^{\circ}$ & RT & {$[\mathbf{M}-\mathbf{H}]^{-}$} & TM & MM & MT & Compounds Identification \\
\hline 12 & 16.47 & 407.0539 & 408.0617 & $\mathrm{C}_{19} \mathrm{H}_{17} \mathrm{O}_{8} \mathrm{Cl}$ & $\mathrm{C}$ & Chloroatranorin \\
\hline 13 & 17.57 & 277.2175 & 278.2203 & $\mathrm{C}_{18} \mathrm{H}_{30} \mathrm{O}_{2}$ & A & $\begin{array}{l}\text { Octadeca-9,12,15-trienoic acid } \\
\text { (linolenelaidic acid) }\end{array}$ \\
\hline 14 & 17.77 & 265.1480 & 266.1558 & $\mathrm{C}_{15} \mathrm{H}_{22} \mathrm{O}_{4}$ & A & $(4 E, 6 E, 9 E)$-Pentadeca-4,6,9-trienedioic acid \\
\hline 15 & 18.03 & 253.2331 & 254.2249 & $\mathrm{C}_{16} \mathrm{H}_{30} \mathrm{O}_{2}$ & A & Palmitoleic acid \\
\hline 16 & 18.34 & 241.2173 & 242.2251 & $\mathrm{C}_{15} \mathrm{H}_{30} \mathrm{O}_{2}$ & A & Pentadecanoic acid \\
\hline 17 & 18.50 & 279.0936 & 280.2409 & $\mathrm{C}_{18} \mathrm{H}_{32} \mathrm{O}_{2}$ & A & Linoleic acid \\
\hline 18 & 18.91 & 267.2331 & 268.2409 & $\mathrm{C}_{17} \mathrm{H}_{32} \mathrm{O}_{2}$ & A & cis-9-Heptadecenoic acid (margaroleic acid) \\
\hline 19 & 19.15 & 255.2329 & 256.2407 & $\mathrm{C}_{16} \mathrm{H}_{32} \mathrm{O}_{2}$ & A & Palmitic acid \\
\hline 20 & 19.35 & 459.3271 & 460.3349 & $\mathrm{C}_{25} \mathrm{H}_{48} \mathrm{O}_{7}$ & Unknown & Unknown \\
\hline 21 & 19.51 & 281.2487 & 282.2565 & $\mathrm{C}_{18} \mathrm{H}_{34} \mathrm{O}_{2}$ & A & Oleic acid \\
\hline 22 & 19.75 & 459.3272 & 460.3350 & $\mathrm{C}_{25} \mathrm{H}_{48} \mathrm{O}_{7}$ & A & Methyl glucose isostearate \\
\hline 23 & 19.89 & 269.2488 & 270.2566 & $\mathrm{C}_{17} \mathrm{H}_{34} \mathrm{O}_{2}$ & A & Heptadecanoic acid (margaric acid) \\
\hline 24 & 19.99 & 307.2645 & 308.2723 & $\mathrm{C}_{20} \mathrm{H}_{36} \mathrm{O}_{2}$ & A & 11,14-Eicosadienoic acid \\
\hline 25 & 20.07 & 457.3722 & 458.3800 & $\mathrm{C}_{27} \mathrm{H}_{54} \mathrm{O}_{5}$ & Unknown & Unknown \\
\hline 26 & 20.18 & 295.2645 & 296.2723 & $\mathrm{C}_{19} \mathrm{H}_{36} \mathrm{O}_{2}$ & A & 10E-nonadecenoic acid \\
\hline 27 & 20.54 & 283.2643 & 284.2721 & $\mathrm{C}_{18} \mathrm{H}_{36} \mathrm{O}_{2}$ & A & Stearic acid (octadecanoic acid) \\
\hline 28 & 20.82 & 309.2800 & 310.2878 & $\mathrm{C}_{20} \mathrm{H}_{38} \mathrm{O}_{2}$ & A & Eicosenoic acid (gondoic acid) \\
\hline 29 & 21.71 & 311.2957 & 312.3035 & $\mathrm{C}_{20} \mathrm{H}_{40} \mathrm{O}_{2}$ & A & Arachidic acid (eicosanoic acid) \\
\hline 30 & 22.62 & 297.1532 & 298.1610 & $\mathrm{C}_{12} \mathrm{H}_{26} \mathrm{O}_{8}$ & Unknown & Unknown \\
\hline 31 & 22.77 & 339.3268 & 340.3346 & $\mathrm{C}_{22} \mathrm{H}_{44} \mathrm{O}_{2}$ & A & Docosanoic acid (behenic acid) \\
\hline 32 & 23.08 & 309.1743 & 310.1821 & $\mathrm{C}_{17} \mathrm{H}_{26} \mathrm{O}_{5}$ & $\mathrm{D}$ & Portentol \\
\hline 33 & 23.58 & 353.2003 & 354.2081 & $\mathrm{C}_{19} \mathrm{H}_{30} \mathrm{O}_{6}$ & Unknown & Unknown \\
\hline 34 & 23.81 & 311.1689 & 312.1767 & $\mathrm{C}_{13} \mathrm{H}_{30} \mathrm{O}_{8}$ & Unknown & Unknown \\
\hline 35 & 23.89 & 367.3579 & 368.3657 & $\mathrm{C}_{24} \mathrm{H}_{48} \mathrm{O}_{2}$ & A & Lignoceric acid (tetracosanoic acid) \\
\hline 36 & 24.01 & 397.2266 & 398.2344 & $\mathrm{C}_{21} \mathrm{H}_{34} \mathrm{O}_{7}$ & $\mathrm{D}$ & Stephanol \\
\hline 37 & 24.60 & 293.1793 & 294.1871 & $\mathrm{C}_{17} \mathrm{H}_{26} \mathrm{O}_{4}$ & A & Heptadecatrienedioic acid \\
\hline 38 & 24.94 & 325.1844 & 326.1922 & $\mathrm{C}_{14} \mathrm{H}_{30} \mathrm{O}_{8}$ & Unknown & Unknown \\
\hline 39 & 25.33 & 395.3895 & 396.3973 & $\mathrm{C}_{26} \mathrm{H}_{52} \mathrm{O}_{2}$ & A & Hexacosanoic acid or cerotic acid \\
\hline 40 & 25.89 & 337.2055 & 338.2133 & $\mathrm{C}_{19} \mathrm{H}_{30} \mathrm{O}_{5}$ & A & 6-Oxononadeca-8,11-dienedioic acid \\
\hline 41 & 26.10 & 339.2000 & 340.2078 & $\mathrm{C}_{15} \mathrm{H}_{32} \mathrm{O}_{8}$ & Unknown & Unknown \\
\hline 42 & 26.24 & 381.2317 & 382.2395 & $\mathrm{C}_{21} \mathrm{H}_{34} \mathrm{O}_{6}$ & A & 19-Acetoxylichesterinic acid \\
\hline 43 & 26.75 & 425.2581 & 426.2659 & $\mathrm{C}_{23} \mathrm{H}_{38} \mathrm{O}_{7}$ & E & Asebotoxin I \\
\hline 44 & 27.53 & 321.2106 & 322.2184 & $\mathrm{C}_{19} \mathrm{H}_{30} \mathrm{O}_{4}$ & A & Nonadecatrienedioic acid \\
\hline 45 & 27.85 & 304.9143 & 305.9221 & Unknown & Unknown & Unknown \\
\hline
\end{tabular}

RT $=$ retention time; $\mathrm{TM}=$ theoretical mass $(\mathrm{m} / \mathrm{z}) ;[\mathrm{M}-\mathrm{H}]^{-}=$measured mass as negative ion $(\mathrm{m} / z) ; \mathrm{MM}=$ molecular mass; $\mathrm{MT}=$ metabolite type; $\mathrm{A}=$ paraconic and aliphatic acids; $\mathrm{B}=$ phenolic compounds; $\mathrm{C}=$ depsides; $\mathrm{D}=$ cycloaliphatic compounds; $\mathrm{E}=$ diterpenes.

Twenty-nine paraconic and aliphatic acids were identified: peaks 1-2, 5-7, 9, 13-19, 2124, 26-29, 31, 35, 37, 39, 40, 42, and 44 using UHPLC-ESI-MS-MS analysis [13,18]. Among these compounds is fumaric acid (Figure S2), a valuable compound used in food, beverages, detergents, animal feed, pharmaceuticals, and various industrial products [85-88]. 
Similarly, for traumatic acid, a phytohormone belongs to the class of cytokinins, a study has demonstrated its positive influence on oxidative stress parameters in normal human fibroblasts [89]. It is also effective against breast cancer cells and has potential anticancer properties and tumour prevention activity. Traumatic acid leads to decreased cell proliferation and viability, GSH/GSSG ratio, and thiol group content. It increased caspase activity, membrane lipid peroxidation, and ROS content, simultaneously reducing breast cancer cell growth through the influence of oxidative stress on apoptosis [90].

Three phenolic compounds were identified and assigned to peaks 4,8 , and 10 using UHPLC-ESI-MS-MS. Peak 4 and peak 10 were identified as p-Coumaric acid 6,7Dihydroxycoumarin (Esculetin) (Figure S2) whose molecular anions were at $m / z 163.0395$ and 177.0186, respectively. To our knowledge, this is the first time that compounds such as these have been found in lichen material. They possess diverse biological and pharmacological properties, including anti-asthma, anti-inflammatory, anti-nociceptive, antioxidative, antitumor, and antiviral activities [91-97]. Gingerol, a phenolic compound (Figure S2), has been identified in the extract WA-hex. Its peak 8 would correspond to $[\mathrm{M}-\mathrm{H}]^{-}$ion at 293.1762 (Table 2).

Peak 11 and 12 were identified as depsides: atranorin $\left([\mathrm{M}-\mathrm{H}]^{-}\right.$ion at $m / z$ 373.0929) and chloroatranorin $\left([\mathrm{M}-\mathrm{H}]^{-}\right.$ion at $m / z$ 407.0539), respectively (Table 2, Figure S2). Atranorin, a derivative of $\beta$-orcinol, is one of the most common secondary metabolites of lichen [98]. It is particularly present in the lichen families Cladoniaceae, Lecanoraceae, Parmeliaceae, and Streocaulaceae [99-101]. In the Physcia genera, there are Physcia caesia [102], Physcia aipolia [103,104], Physcia alnophila [105], and Physcia sorediosa [106]. In recent years, atranorin has been the most extensively studied. Indeed, it has shown antioxidant [107], antimicrobial [108], anti-inflammatory [109], antinociceptive [110], wound healing [111], and photoprotective properties [112]. Additionally, it exerted strong inhibitory effects on cancer cell proliferation, migration, and actin cytoskeleton organisation [113].

Portentol, peak $32\left([\mathrm{M}-\mathrm{H}]^{-}\right.$ion at $m / z$ 309.1743) and Stephanol peak $36([\mathrm{M}-\mathrm{H}]-$ ion at $m / z$ 397.2266), which are cycloaliphatic compounds were also identified in WA-hex (Table 2, Figure S2). Likewise for asebotoxin I, a toxic diterpene has been identified at the peak $43\left([\mathrm{M}-\mathrm{H}]^{-}\right.$ion at $m / z$ 425.2581). It was initially and only discovered in the plant Pieris japonica [114], but our results show the opposite, i.e., that the compound can be detected in lichen species.

\subsubsection{ME-hex Extract Analysis of Physcia mediterranea}

In the present study, the analysis of the phytochemical profile of ME-hex using UHPLCESI-MS/MS, in negative ion mode, resulted in the detection of 54 significant compounds indicated in Figure S3. Only four compounds could not be identified. The identified compounds are of paraconic and aliphatic acids, aromatic polyketides (depsides, depsones, and phenyl ethers), phenolic acids, sesquiterpenes lactones, triterpenes, carboxybenzaldehyde, and carboxyphthalide types (Table 3).

Table 3. Identification of metabolites in ME-hex of Physcia mediterranea by UHPLC/ESI/MS/MS.

\begin{tabular}{cccccccc}
\hline $\mathbf{N}^{\circ}$ & $\mathbf{R T}$ & $\mathbf{M}-\mathbf{H}]^{-}$ & $\mathbf{T M}$ & $\mathbf{M M}$ & $\mathbf{M T}$ & Compounds & MS ${ }^{2}$ Ions \\
\hline 1 & 2.72 & 187.0970 & 188.1043 & $\mathrm{C}_{9} \mathrm{H}_{16} \mathrm{O}_{4}$ & $\mathrm{~A}$ & 3,5-Dimethoxyciclohexanecarboxylic acid & $\begin{array}{c}141.8670 ; \\
123.0807\end{array}$ \\
\hline 2 & 2.75 & 293.0669 & 294.0741 & $\mathrm{C}_{14} \mathrm{H}_{14} \mathrm{O}_{7}$ & $\mathrm{~A}$ & $\begin{array}{c}\text { 6-(Hydroxymethyl)-3,5-bis(methoxycarbonyl)- } \\
\text { 2,4-dimethylcyclohex-1-ene-1-carboxylic acid }\end{array}$ & $\begin{array}{c}234.0530 ; \\
207.0695\end{array}$ \\
\hline 3 & 3.08 & 243.1239 & 244.1311 & $\mathrm{C}_{12} \mathrm{H}_{20} \mathrm{O}_{5}$ & A & $\begin{array}{r}\text { 3,5,6-Hydroxymethyl-2,4-dimethylcyclohex-1- } \\
\text { ene-1-carboxylic acid }\end{array}$ & $\begin{array}{c}183.1025 ; \\
176.6474\end{array}$ \\
\hline 4 & 3.15 & 151.0393 & 152.0465 & $\mathrm{C}_{8} \mathrm{H}_{8} \mathrm{O}_{3}$ & $\mathrm{~B}$ & Atranol & $\begin{array}{c}123.0444 ; \\
105.0335 ; \\
81.0335\end{array}$ \\
\hline
\end{tabular}


Table 3. Cont.

\begin{tabular}{|c|c|c|c|c|c|c|c|}
\hline $\mathbf{N}^{\circ}$ & RT & {$[\mathbf{M}-\mathbf{H}]^{-}$} & TM & MM & MT & Compounds & MS $^{2}$ Ions \\
\hline 5 & 3.20 & 225.1129 & 226.1201 & $\mathrm{C}_{12} \mathrm{H}_{18} \mathrm{O}_{4}$ & A & $\begin{array}{l}\text { 5-Formyl-3-hydroxymethyl-2,4,6- } \\
\text { trimethylcyclohex-1-ene-1-carboxylic acid }\end{array}$ & $\begin{array}{c}\text { 207.1023; } \\
97.0284\end{array}$ \\
\hline 6 & 3.47 & 199.0973 & 200.1046 & $\mathrm{C}_{10} \mathrm{H}_{16} \mathrm{O}_{4}$ & $\mathrm{~A}$ & $\begin{array}{l}\text { 3,5-Dihydroxy-2,4,6- } \\
\text { trimethylciclohexenecarboxilic acid }\end{array}$ & $\begin{array}{l}183.4492 \\
162.8385 \\
114.9508\end{array}$ \\
\hline 7 & 3.58 & 149.0237 & 150.0310 & $\mathrm{C}_{8} \mathrm{H}_{6} \mathrm{O}_{3}$ & $\mathrm{C}$ & 4-Formylbenzoic acid & - \\
\hline 8 & 3.82 & 241.1081 & 242.1153 & $\mathrm{C}_{12} \mathrm{H}_{18} \mathrm{O}_{5}$ & A & $\begin{array}{l}\text { 5-Formyl-3,6-dihydroxymethyl-2,4- } \\
\text { dimethylcyclohex-1-enecarboxylic acid }\end{array}$ & $\begin{array}{r}218.816 \\
172.6485\end{array}$ \\
\hline 9 & 4.10 & 201.1129 & 202.1202 & $\mathrm{C}_{10} \mathrm{H}_{18} \mathrm{O}_{4}$ & $\mathrm{~A}$ & $\begin{array}{c}\text { 2,4-Dihydroxy-3,5,6-trimethylcyclohexane-1- } \\
\text { carboxylic acid }\end{array}$ & $\begin{array}{l}197.6263 ; \\
164.8350 \\
139.1124\end{array}$ \\
\hline 10 & 4.95 & 199.1337 & 200.1409 & $\mathrm{C}_{11} \mathrm{H}_{20} \mathrm{O}_{3}$ & A & 2-Hydroxy-10-undecenoic acid & - \\
\hline 11 & 5.00 & 185.0006 & 186.0079 & $\mathrm{C}_{8} \mathrm{H}_{7} \mathrm{ClO}_{3}$ & $\mathrm{~B}$ & Chloroatranol & - \\
\hline 12 & 5.21 & 169.0863 & 170.0936 & $\mathrm{C}_{9} \mathrm{H}_{14} \mathrm{O}_{3}$ & A & $\begin{array}{l}\text { 4-Hydroxy-2,5-dimethylcyclohex-1-ene-1- } \\
\text { carboxylic acid }\end{array}$ & $\begin{array}{l}124.9796 ; \\
104.4180\end{array}$ \\
\hline 13 & 5.49 & 163.0392 & 164.0470 & $\mathrm{C}_{9} \mathrm{H}_{8} \mathrm{O}_{3}$ & $\mathrm{D}$ & $p$-Coumaric acid & - \\
\hline 14 & 5.60 & 209.1181 & 210.1253 & $\mathrm{C}_{12} \mathrm{H}_{18} \mathrm{O}_{3}$ & A & $\begin{array}{l}\text { 6-(1-Oxopentyl)-1-cyclohexene-1- } \\
\text { carboxylic acid }\end{array}$ & $\begin{array}{l}146.9850 ; \\
105.0336\end{array}$ \\
\hline 15 & 5.87 & 215.1286 & 216.1359 & $\mathrm{C}_{11} \mathrm{H}_{20} \mathrm{O}_{4}$ & A & Undecanedioic acid & - \\
\hline 16 & 6.57 & 227.1288 & 228.1360 & $\mathrm{C}_{12} \mathrm{H}_{20} \mathrm{O}_{4}$ & A & trans-Dodec-2-enedioic acid & - \\
\hline 17 & 7.44 & 282.2078 & 283.2150 & $\mathrm{C}_{16} \mathrm{H}_{29} \mathrm{NO}_{3}$ & A & $N$-Dodecanoyl-L-Homoserine lactone & - \\
\hline 18 & 9.53 & 243.1601 & 244.1675 & $\mathrm{C}_{13} \mathrm{H}_{24} \mathrm{O}_{4}$ & A & Tridecanedioic acid & - \\
\hline 19 & 10.59 & 311.2230 & 312.2302 & $\mathrm{C}_{18} \mathrm{H}_{32} \mathrm{O}_{4}$ & A & 9Z-Octadecenedioic acid & - \\
\hline 20 & 10.87 & 233.1547 & 234.1619 & $\mathrm{C}_{15} \mathrm{H}_{22} \mathrm{O}_{2}$ & E & Fukinanolide & - \\
\hline 21 & 10.89 & 323.2230 & 324.2303 & $\mathrm{C}_{19} \mathrm{H}_{32} \mathrm{O}_{4}$ & $\mathrm{~F}$ & allo-Protolichesterinic acid & - \\
\hline 22 & 12.28 & 313,2388 & 314.2266 & $\mathrm{C}_{18} \mathrm{H}_{34} \mathrm{O}_{4}$ & A & Octadecanedioic acid & - \\
\hline 23 & 13.04 & 389.1245 & 390.1315 & $\mathrm{C}_{20} \mathrm{H}_{22} \mathrm{O}_{8}$ & B & 8-Hydroxydiffractaic acid & - \\
\hline 24 & 13.34 & 293.2124 & 294.2202 & $\mathrm{C}_{18} \mathrm{H}_{30} \mathrm{O}_{3}$ & A & 2-Hydroxylinolenic acid & - \\
\hline 25 & 13.58 & 291.1968 & 292.2041 & $\mathrm{C}_{18} \mathrm{H}_{28} \mathrm{O}_{3}$ & A & $\alpha$-Licanic acid & - \\
\hline 26 & 14.38 & 295.2279 & 296.2351 & $\mathrm{C}_{18} \mathrm{H}_{32} \mathrm{O}_{3}$ & A & 2-Hydroxylinoleic acid & - \\
\hline 27 & 14.40 & 295,2278 & 296,2352 & $\mathrm{C}_{18} \mathrm{H}_{32} \mathrm{O}_{3}$ & A & 18-hydroxylinoleic acid & - \\
\hline 28 & 15.19 & 297.2436 & 298.2508 & $\mathrm{C}_{18} \mathrm{H}_{34} \mathrm{O}_{3}$ & A & 9-Oxooctadecanoic acid & - \\
\hline 29 & 15.25 & 295.2280 & 296.2351 & $\mathrm{C}_{18} \mathrm{H}_{32} \mathrm{O}_{3}$ & A & Coriolic acid & - \\
\hline 30 & 15.36 & 297.2435 & 298.2508 & $\mathrm{C}_{18} \mathrm{H}_{34} \mathrm{O}_{3}$ & A & Ricinoleic acid & - \\
\hline 31 & 15.43 & 177.0187 & 178.0259 & $\mathrm{C}_{9} \mathrm{H}_{6} \mathrm{O}_{4}$ & $\mathrm{D}$ & 6,7-Dihydroxycoumarin & - \\
\hline 32 & 15.46 & 365.2330 & 366.2403 & $\mathrm{C}_{21} \mathrm{H}_{34} \mathrm{O}_{5}$ & G & Muronic acid & - \\
\hline 33 & 16.37 & 471.3481 & 472.3553 & $\mathrm{C}_{30} \mathrm{H}_{48} \mathrm{O}_{4}$ & Unknown & Unknown & - \\
\hline 34 & 16.47 & 210.9834 & 211.9873 & $\mathrm{C}_{9} \mathrm{H}_{5} \mathrm{ClO}_{4}$ & $\mathrm{H}$ & $\begin{array}{l}\text { 7-chloro-3-oxo-1,3-dihydroisobenzofuran-5- } \\
\text { carboxylic acid }\end{array}$ & $\begin{array}{l}138.9948 \\
103.0180\end{array}$ \\
\hline 35 & 16.51 & 407.0540 & 408.0611 & $\mathrm{C}_{19} \mathrm{H}_{17} \mathrm{ClO}_{8}$ & B & Chloroatranorin & - \\
\hline 36 & 17.59 & 387.2544 & 388.2616 & $\mathrm{C}_{24} \mathrm{H}_{36} \mathrm{O}_{4}$ & Unknown & Unknown & - \\
\hline 37 & 17.68 & 389.1242 & 390.1314 & $\mathrm{C}_{20} \mathrm{H}_{22} \mathrm{O}_{8}$ & I & Leprolomin & - \\
\hline 38 & 18.04 & 253.2173 & 254.2244 & $\mathrm{C}_{16} \mathrm{H}_{30} \mathrm{O}_{2}$ & A & Palmitoleic acid & - \\
\hline
\end{tabular}


Table 3. Cont.

\begin{tabular}{|c|c|c|c|c|c|c|c|}
\hline $\mathbf{N}^{\circ}$ & RT & {$[\mathbf{M}-\mathbf{H}]^{-}$} & TM & MM & MT & Compounds & MS $^{2}$ Ions \\
\hline 39 & 18.33 & 241.2172 & 242.2245 & $\mathrm{C}_{15} \mathrm{H}_{30} \mathrm{O}_{2}$ & $\mathrm{~A}$ & Pentadecanoic acid & - \\
\hline 40 & 18.35 & 455.3531 & 456.3605 & $\mathrm{C}_{30} \mathrm{H}_{48} \mathrm{O}_{3}$ & G & Ursolic acid & - \\
\hline 41 & 18.50 & 279.2330 & 280.2403 & $\mathrm{C}_{18} \mathrm{H}_{32} \mathrm{O}_{2}$ & A & Linoleic acid & - \\
\hline 42 & 18.66 & 299.2595 & 300.2667 & $\mathrm{C}_{18} \mathrm{H}_{36} \mathrm{O}_{3}$ & A & 2-Hydroxyoctadecanoic acid & - \\
\hline 43 & 18.78 & 279.2332 & 280.2403 & $\mathrm{C}_{18} \mathrm{H}_{32} \mathrm{O}_{2}$ & A & Linoleic acid & - \\
\hline 44 & 19.16 & 255.2329 & 256.2401 & $\mathrm{C}_{16} \mathrm{H}_{32} \mathrm{O}_{2}$ & A & Palmitic acid & - \\
\hline 45 & 19.49 & 281.2485 & 282.2559 & $\mathrm{C}_{18} \mathrm{H}_{34} \mathrm{O}_{2}$ & A & Oleic acid & - \\
\hline 46 & 19.62 & 269.2488 & 270.2561 & $\mathrm{C}_{17} \mathrm{H}_{34} \mathrm{O}_{2}$ & A & 15-Methylhexadecanoic acid & - \\
\hline 47 & 19.89 & 269.2488 & 270.2561 & $\mathrm{C}_{17} \mathrm{H}_{34} \mathrm{O}_{2}$ & A & Heptadecanoic acid & - \\
\hline 48 & 20.07 & 327.2543 & 328.2616 & $\mathrm{C}_{20} \mathrm{H}_{40} \mathrm{O}_{3}$ & A & 2-Hydroxyeicosanoic acid & - \\
\hline 49 & 20.54 & 283.2643 & 284.2716 & $\mathrm{C}_{18} \mathrm{H}_{36} \mathrm{O}_{2}$ & A & Stearic acid (octadecanoic acid) & - \\
\hline 50 & 20.74 & 309.2801 & 310.2875 & $\mathrm{C}_{20} \mathrm{H}_{38} \mathrm{O}_{2}$ & A & Eicosenoic acid (Gondoic acid) & - \\
\hline 51 & 21.14 & 297.2801 & 298.2873 & $\mathrm{C}_{19} \mathrm{H}_{38} \mathrm{O}_{2}$ & A & Nonadecanoic acid & - \\
\hline 52 & 21.70 & 311.2957 & 312.3029 & $\mathrm{C}_{20} \mathrm{H}_{40} \mathrm{O}_{2}$ & A & Arachidic acid (Eicosanoic acid) & - \\
\hline 53 & 22.68 & 637.4841 & 638.4908 & $\mathrm{C}_{41} \mathrm{H}_{66} \mathrm{O}_{5}$ & Unknown & Unknown & - \\
\hline 54 & 24.51 & 605.3483 & $\begin{array}{r}606 . \\
3561\end{array}$ & $\mathrm{C}_{37} \mathrm{H}_{50} \mathrm{O}_{7}$ & Unknown & Unknown & - \\
\hline
\end{tabular}

$\mathrm{RT}=$ retention time $(\mathrm{min}) ; \mathrm{TM}=$ theoretical mass $(\mathrm{m} / \mathrm{z}) ;[\mathrm{M}-\mathrm{H}]^{-}=$measured mass as negative ion $(m / z) ; \mathrm{MM}=\mathrm{molecular}$ mass; $\mathrm{MT}=$ metabolite type; $\mathrm{A}=$ paraconic and aliphatic acids; $\mathrm{B}=$ depsides; $\mathrm{C}=$ carboxybenzaldehyde; $\mathrm{D}=$ phenolic acids; $\mathrm{E}=$ sesquiterpene lactones; $\mathrm{F}$ = depsones; $\mathrm{G}=$ triterpene; $\mathrm{H}$ = carboxyphthalide; $\mathrm{I}=$ diphenyl ether.

Thirty-seven paraconic and aliphatic acids corresponding to peaks 1-3, 5, 6, 8-10, 12, 14-19, 22, 24-30, 38, 39, and 41-52 were identified using UHPLC/ESI/MS/MS analysis. 3,5-Dimethoxyciclohexanecarboxylic acid (Figure 4) was identified as peak 1 (molecular anion at $m / z 188.1043)$. The fragmentation of peak 1 produced ion at $m / z 143.8651$ [M $\left.\mathrm{H}-\mathrm{CO}_{2}\right]^{-}$, and $141.8670\left[\mathrm{M}-\mathrm{H}-\mathrm{CO}_{2}\right]^{-}$. Peak 2, with an $[\mathrm{M}-\mathrm{H}]^{-}$ion at $m / z 294.0741$, was identified as 6-(hydroxymethyl)-3,5-bis(methoxycarbonyl)-2,4-dimethylcyclohex-1ene-1-carboxylic acid (Figure 4). The fragmentation of peak 1 produced ion at $\mathrm{m} / z 249.0771$ $\left[\mathrm{M}-\mathrm{H}-\mathrm{CO}_{2}\right]^{-}, 234.0530\left[\mathrm{M}-\mathrm{H}-\mathrm{CO}_{2}-\mathrm{CH}_{3}\right]^{-}$, and $207.0695\left[\mathrm{M}-\mathrm{H}-\mathrm{CO}_{2}\right.$ $\left.-\mathrm{CH}_{3}\right]^{-}$, confirming this compound. Peak 3 was identified as 3,5,6-hydroxymethyl2,4-dimethylcyclohex-1-ene-1-carboxylic acid (Figure 4), which showed a $[\mathrm{M}-\mathrm{H}]^{-}$at $m / z$ 244.1311. Major diagnostic daughter MS ions of the compound were $[\mathrm{M}-\mathrm{H}-$ $\left.\mathrm{CO}-\mathrm{CH}_{3} \mathrm{OH}\right]^{-}$and $\left[\mathrm{M}-\mathrm{H}-\mathrm{H}_{2} \mathrm{O}_{2}\right]^{-}$(183.1025 and 176.6474 a.m.u, respectively). Peak 5 with a $[\mathrm{M}-\mathrm{H}]^{-}$ion at $m / z 226.1201$ was identified as 5-formyl-3-hydroxymethyl-2,4,6trimethylcyclohex-1-ene-1-carboxylic acid (Figure 4). The fragmentation of the peak produced ions at $m / z 207.1023$ and 97.0284 confirming this structure. Peak 6 was identified as 3,5-dihydroxy-2,4,6-trimethylciclohexenecarboxilic acid (molecular anion at $\mathrm{m} / \mathrm{z} 200.1046$ ) (Figure 4). Major daughter ions of peak 6 were at $m / z$ 183.4492, 162.8385, and 114.9508. Peak 8 with a $[\mathrm{M}-\mathrm{H}-]^{-}$ion at $m / z 242.1153$ was identified as 4-O-demethylbaeomycesic acid, and their major diagnostic daughter $\mathrm{MS}$ ions were $\left[\mathrm{M}-\mathrm{H}-\mathrm{CO}_{2}\right]^{-}$and $[\mathrm{M}-$ $\left.\mathrm{H}-2 \mathrm{CH}_{3} \mathrm{OH}\right]^{-}$, (218.8167 and 172.6485 a.m.u, respectively). The 2,4-dihydroxy-3,5,6trimethylcyclohexane-1-carboxylic acid (Figure 4 ) was identified as Peak $8\left([\mathrm{M}-\mathrm{H}]^{-}\right.$at $m / z$ 202.1202). The fragmentation of this compound produced three ions at $m / z 197.6263$, 164.8350 and 139.1124 confirming its structure. Peak 12 was identified as 4-hydroxy-2,5dimethylcyclohex-1-ene-1-carboxylic acid (Figure 4), which showed a $[\mathrm{M}-\mathrm{H}]^{-}$peak at $m / z$ 170.0936. Major diagnostic daughter $\mathrm{MS}$ ions were $[\mathrm{M}-\mathrm{H}-\mathrm{CO}]^{-},\left[\mathrm{M}-\mathrm{H}-\mathrm{CO}_{2}\right]^{-}$, and $\left[\mathrm{M}-\mathrm{H}-\mathrm{CO}_{2}\right]^{-}(193.0514,140.9478,124.9796$, and 104.4180 a.m.u., respectively). Peak 14 was assigned to 6-(1-Oxopentyl)-1-cyclohexene-1-carboxylic acid (Figure 4) on its 
resolution molecular anion at $m / z 210.1253\left([\mathrm{M}-\mathrm{H}]^{-}\right.$peak). It produced major diagnostic MS ions at 164.8947, 146.9850, and 105.0336.<smiles>CC1=C(C(=O)O)C(CO)C(C=O)C(C)C1C=O</smiles>

(3)<smiles>CC1=C(C(=O)O)C(C=O)C(C=O)C(C)C1C=O</smiles>

(8)<smiles>Cc1cc(O)c(C=O)c(O)c1</smiles>

(4)<smiles>CC1C(C)C(O)C(C(=O)O)C(O)C1C</smiles>

(9)<smiles>CC1=C(C(=O)O)C(C)C(C=O)C(C)C1CO</smiles>

(5)<smiles>CC1=C(C(=O)O)CC(C)C(O)C1</smiles>

(12)<smiles>CC1=C(C(=O)O)C(O)C(C)C(O)C1C</smiles>

(6)<smiles>O=C(O)c1cc(Cl)c2c(c1)C(=O)OC2</smiles><smiles>CCCCC(=O)C1CCCC=C1C(=O)O</smiles>

(14)

Figure 4. Chemical structure of Compound 3, 4, 5, 6, 8, 9, 12, 14, and 38 identified in the ME-hex of Physcia mediterranea by UHPLC/ESI/MS/MS.

According to the results (Table 3), four depsides compounds were identified corresponding to peak 4, 11, 23, and 35 in the ME-hex extract. Peak 4 was identified as atranol (Figure 5) (molecular anion at $\mathrm{m} / z$ 152.0465). The fragmentation of peak 4 also produced ions at $123.0444\left[\mathrm{M}-\mathrm{H}-\mathrm{CO}_{2}\right]^{-}, 105.0335\left[\mathrm{M}-\mathrm{H}-\mathrm{CO}_{2}-\mathrm{H}_{2} \mathrm{O}_{2}\right]^{-}$, and $81.0335[\mathrm{M}-$ $\left.\mathrm{H}-\mathrm{CO}_{2}-\mathrm{H}_{2} \mathrm{O}_{2}\right]^{-}$confirming this compound. Chloroatranol and 8-Hydroxydiffractaic acid identified at peak 11 and 23, showed $[\mathrm{M}-\mathrm{H}]^{-}$ions at $\mathrm{m} / z 186.0079$ and 390.1315, respectively. This is also chloratranorin (Figure 5) at peak 35 , which shows $[\mathrm{M}-\mathrm{H}]^{-}$ions at $m / z 408.0611$.

A despsone named allo-protolichesterinic acid was also detected in ME-hex extract. It would correspond to the peak 21 and shows $[\mathrm{M}-\mathrm{H}]^{-}$ions at $m / z$ 324.2303. Moreover, two phenolic acids were identified, corresponding to peak 13 and 31: p-Coumaric acid and dihydroxycoumarin (Figure 5). They showed [M - H] $]^{-}$ions at $m / z 164.0470$ and 178.0259 respectively. Peak 34 was identified as 7-chloro-3-oxo-1,3-dihydroisobenzofuran5-carboxylic acid (carboxyphthalide) (Figure 5), which showed a $[\mathrm{M}-\mathrm{H}]^{-}$peak at $m / z$ 211.9873. It produced major diagnostic MS ions at $m / z 138.9948$ and 103.0180, confirming this compound. 
<smiles>COc1ccc(CCOC(C)(C)O)cc1</smiles>

(7)<smiles>Cc1cc(O)c(C=O)c(O)c1Cl</smiles>

(11)<smiles>O=C(O)C=Cc1ccc(O)cc1</smiles>

(13)

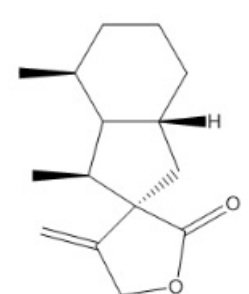

(20)<smiles>O=C1CCc2cc(O)c(O)cc2O1</smiles>

(31)<smiles>C=C1C(=O)O[C@@H](CCCCCCCCCCCCCC(C)=O)C1C(=O)O</smiles>

(32)<smiles>C[C@H]1CC[C@]2(C(=O)O)CC[C@]3(C)C(=CC[C@@]4(C)[C@@]3(C)CC[C@]3(C)C(C)(C)[C@@H](O)CC[C@@]34C)[C@]2(C)[C@H]1C</smiles><smiles>COc1cc(Oc2c(O)c(C)c(OC)c(C(C)=O)c2O)c(C(C)=O)c(O)c1C</smiles>

(37)

Figure 5. Chemical structure of compound 7, 11, 13, 20, 23, 31, 32, 37, and 39 identified in ME-hex of Physcia mediterranea by UHPLC/ESI/MS/MS.

It is similar for the compounds fukinanolide (sesquiterpene lactones) at peak 20 and leprolomin (diphenyl ether) (Figure 5) at peak 37. They showed a $[\mathrm{M}-\mathrm{H}]^{-}$peak at $m / z$ 312.2302 and 366.2403 respectively. In the study by Zhang et al. (2016), fukinanolide, also called bakkenolide A, extracted from the plant Petasites tricholobus, showed antiinflammatory properties in the treatment of leukaemia [115]. In the lichens group, the sesquiterpene has only been identified in Cetraria islandica [116].

Finally, two triterpenes, muronic acid at peak 32 and ursolic acid at peak 39, were also identified (Figure 5) and produced major diagnostic MS ions at $m / z$ 390.1314, 456.3605. Muronic acid was previously identified in Usnea rubicunda, Usnea subfloridana [117], and Punctelia microsticta [118]. On the other hand, ursolic acid (3 $\beta$-hydroxy-urs-12-ene-28-oic acid) is widespread in the vegetable kingdom [119]. The lichenic species Ramalina hierrensis [16], Ramalina hierrensis [120], Stereocaulon evolutum [121], and Pannaria tavaresii [122] also contain this compound.

\subsection{NMR Analysis}

$1 \mathrm{H}$ and C13 NMR analysed the two samples from WA-hex and ME-hex. The results we obtained reveal the predominance of the secondary metabolite atranorin only in the WA-hex sample. The structure has been characterised, and the NMR spectra are compared with those of a previously isolated sample of Physcia sorediosa [106,123], demonstrated in Figures S4 and S5.

Atranorin (Figure 6) is the most common secondary metabolite in lichens and is mainly found on lichens' surface (cortex) [124]. It acts as a photo-buffer because it reflects harmful 
UV rays to the thallus's surface and allows the lichens to live in areas receiving intense solar radiation [125].<smiles>COC(=O)c1c(C)cc(OC(=O)c2c(C)cc(O)c(C=O)c2O)c(F)c1O</smiles>

Figure 6. Chemical structure of atranorin isolated from WA-hex of Physcia mediterranea.

Several factors can influence atranorin concentrations in lichens. They fluctuate with the seasons [126,127] and vary according to the habitat [128]. The method of preparation and extraction of lichens can also influence the concentration of this metabolite. Conventional organic solvents (such as hexane and acetone) are commonly used for its extraction as hydrophobic [129]. Our study used n-hexane with Soxhlet extraction method, which is of choice in studying organic analytes extracted from lichens [130]. It is still used to date to extract organic air pollutants, organochlorinated pesticides and insecticides from the lichen matrix [131,132].

According to Komaty et al. (2015), the lichen grinding method can affect the extraction efficiency and even be used to selectively increase the extraction efficiency of certain secondary metabolites such as atranorin [133]. To achieve a higher yield, use a blender instead of a ball mill, as it selectively grinds the cortex into a fine powder, which can be recovered from the larger medulla pieces. A ball mill or a mortar and pestle technique will reduce the whole lichen to powder, which will reduce the extraction efficiency of the atranorin [129]. Pseudevernia furfuracea is a lichen widely used as a raw material in the perfume and cosmetics industries due to its richness in aromatic compounds [33,134-137]. Microwave-assisted extraction of this lichen has increased atranorin extraction efficiency by a factor of five [133].

\section{Materials and Methods}

\subsection{Lichen Material}

The saxicolous lichen specimen Physcia mediterranea Nimis was collected at Ain Tebib (Sector Oum tboul) on the rock, at an altitude of $120 \mathrm{~m}$ above sea level, coordinate $36^{\circ} 49^{\prime} 09^{\prime \prime} \mathrm{N} ; 08^{\circ} 31^{\prime} 33^{\prime \prime} \mathrm{E}$ in June 2017 (Figure 7). Ain Tebib station is located in the national park of El Kala $(80,000$ ha). The collected lichen samples were packed in polyethene bags and stored at $4{ }^{\circ} \mathrm{C}$ until further processed. Professor Monia Ali Ahmed has identified Physcia mediterranea Nimis (Figure 1), lichenologist and research director of the Pathology of Ecosystems team at the University of Badji-Mokhtar, Annaba, Algeria. Botanical description of Physcia mediterranea Nimis is in Figure S6. The identification was confirmed by Pr Jean Michel Sussey, lichenologist at the French Association of Lichenology (AFL). This sample has been deposited in Badji-Mokhtar University, Annaba, code AAM-1. 


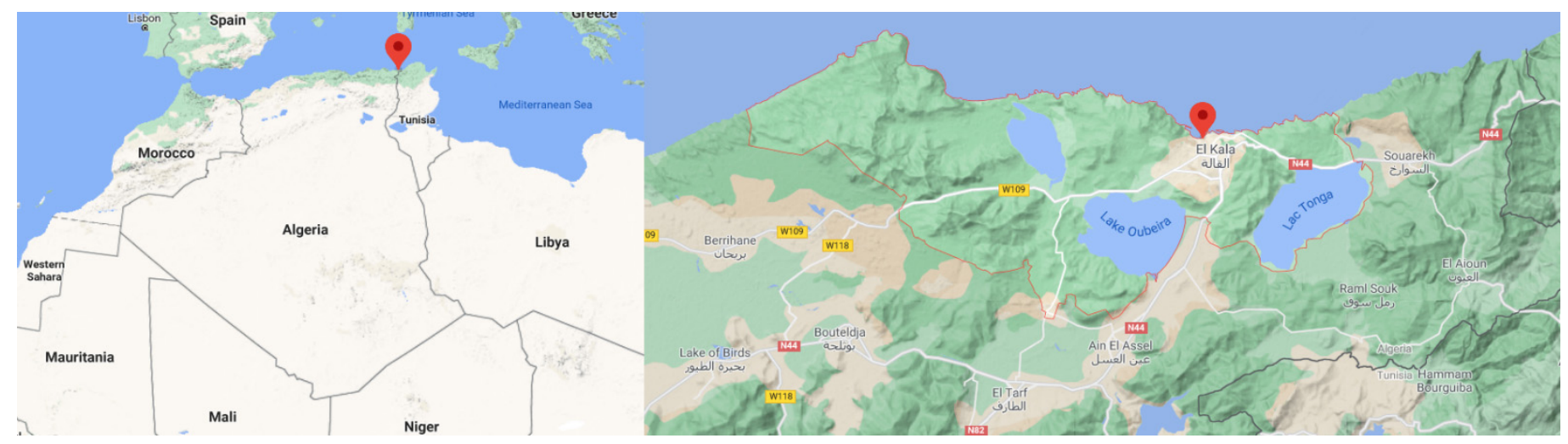

Figure 7. The topography of Algeria, indicating El Kala region boundaries $\left(36^{\circ} 49^{\prime} 09^{\prime \prime} \mathrm{N} ; 08^{\circ} 31^{\prime} 33^{\prime \prime} \mathrm{E}\right)$.

\subsection{Sample Preparation}

The lichens were washed with tap water to remove the dust and other foreign materials. The washed samples were dried under shade for a week. The lichen was ground using a grinder. The preparations were then pulverised into powdered form by using heavyduty blender.

\subsection{Preparation of Physcia mediterranea Extracts}

The powder samples ( $24 \mathrm{~g}$ ) of Physcia mediterranea Nimis were extracted with the solvent n-hexane $(500 \mathrm{~mL})$ using a Soxhlet extractor $24 \mathrm{~h}$. After complete extraction, the solvent was evaporated using a rotary evaporator under reduced pressure to obtain nhexane extract $(1.026 \mathrm{~g})$. It was then extracted with hot methanol $\left(60^{\circ} \mathrm{C}\right)$ to obtain two parts: insoluble precipitate representing lichen wax (WA-hex) and the methanol soluble part (ME-hex). Both extracts (WA-hex/ME-hex) were completely evaporated using a rotary evaporator under reduced pressure to obtain dry extracts $(0.552 \mathrm{~g} / 0.300 \mathrm{~g})$, respectively.

\subsection{Instrumentation and Analysis Parameters}

In this present study, the chemical composition of the two fractions WA-hex and ME-hex of Algerian Physcia mediterranea Nimis was analysed using the HPLC-ESI-MS-MS method. Moreover, we combined GC-MS's ability with the targeted metabolomics of HPLC-ESI-MS-MS methods to characterise the composition of n-hexane extract (ME-hex) for the first time. In addition, most compounds potentially present in the wax (WA-hex) are detected and characterised using the NMR method.

\subsubsection{GC-MS Analysis}

For the GC-MS analysis, an Agilent MS220 (Varian, Inc. Walnut Creek, CA, USA) mass spectrometer coupled to a 7890A GC. The oven temperature was initially set to $50{ }^{\circ} \mathrm{C}$, held for $5 \mathrm{~min}$, then a ramp of $30^{\circ} \mathrm{C} / \mathrm{min}$ was applied up to $270{ }^{\circ} \mathrm{C}$ that was held for an additional5 min. MS spectra were acquired in EI mode with a mass range from 50 to 600 a.m.u. Before being injected into the GC-MS system, the ME-hex fraction was pre-esterified with diazomethane, in order to identify eventual less polar compounds in this fraction. It was then solubilised in dichloromethane and injected into the apparatus.

\subsubsection{NMR Analysis}

Proton $1 \mathrm{H}$ and carbon 13C NMR spectroscopy were recorded on a Brüker Advance III $400 \mathrm{MHz}$ spectrometer (Brüker Scientific Inc, Billerica, MA, USA) at $400 \mathrm{MHz}$ for proton and $100 \mathrm{MHz}$ for carbon. The recovered WA was dissolved in deuterated solvent's $\left(\mathrm{CDCl}_{3}\right)$ $(5 \mathrm{mg} / \mathrm{mL})$, at room temperature. The solution was transferred to $5 \mathrm{~mm}$ outside diameter tubes, and the spectra were acquired at room temperature. The deuterated solvent's residual peak signal was for $1 \mathrm{H}$ spectra at $7.26 \mathrm{ppm}$ and $13 \mathrm{C}$ spectra at $77.2 \mathrm{ppm}$. The 
chemical deviations $(\delta)$ are expressed in parts per million (ppm) and the coupling constants (J) in Hertz. The data was processed using TOPSPIN 3.5 software (Brüker Scientific Inc.).

\subsubsection{HPLC-ESI-MS-MS}

The method was carried out on an orbitrap Thermo q-Exactive mass spectrometer coupled to a Vanquish HPLC. A Kinetex XB-C18 (Phenomenex) with a particle size of 2.6 microns, $100 \mathrm{~mm}$ of length, and a diameter of $2.1 \mathrm{~mm}$ was used as a column. The mobile phases were $0.1 \%$ formic aqueous solution (A) and, acetonitrile (B). The gradient program (time (min), \% B) was: $(0.00,50) ;(20.00,100) ;(25.00,100) ;(26.00,50)$. The flow rate was $0.200 \mathrm{~mL} \mathrm{~min}^{-1}$ and the injection volume was $10 \mu \mathrm{L}$.

The ionisation electrospray in negative mode was used. The following analysis parameters were: electrospray voltage $-3.8 \mathrm{kV}$, sheath gas flow rate, 30 ; auxiliary gas unit flow rate, 10; drying gas temperature, $310{ }^{\circ} \mathrm{C}$; capillary temperature, $320^{\circ} \mathrm{C}$; S-lens and $\mathrm{RF}$ level, 55. The acquisition was performed in a mass range from 100 to 1000 a.m.u. An auto MS2 program was used with a fragmentation voltage of 30 .

\section{Conclusions}

Knowledge of the chemical constituents of lichens is invaluable as this information will be useful for the synthesis of potential new chemical substances. Many researchers report such phytochemical screening of various lichens [103,106,116,117,123,124]. A growing body of evidence indicates that lichens' secondary metabolites play an essential role in human health and may be nutritionally important $[24,26,27,29-31,58,112,118-120]$. In the present study, we have identified and chemically characterised Algerian Physcia mediterranea Nimis for the first time. The extraction of these metabolites was carried out with hot hexane. A methanol-crystallisation process allowed us to characterise and identify the atranorine depside, as a major component of lichen wax. In this work, several aromatic acids, a kaurane, and fatty acids have been identified by GC-MS of ME-hex. The UHPLC-ESI-MSMS technique has been used to analyse the crystallised fraction, WA-hex, whose major product is atranorin and chloroatranorin with the minority products identified. In MEhex fraction, this technique identifies paraconic and aliphatic acids, depsides, depsones, phenolic compounds, sesquiterpenes, triterpenes, and phenyl ethers in addition to other minority derivatives. This study reveals for the first time the different compounds of Physcia medeterranea considered as a rare international species; furthermore, it highlights the importance of the lichens of Algeria as a promising source of bioactive molecules.

Supplementary Materials: The following are available online, Figure S1 (UHPLC/ESI/MS/MS chromatogram of Physcia mediterranea (WA-hex). Figure S2: Chemical structure of Compounds identified in WA-hex of Physcia mediterranea by UHPLC/ESI/MS/MS. Figure S3: UHPLC/ESI/MS/MS chromatogram of Physcia mediterranea (ME-hex). Figure S4: 1H NMR spectrum of WA-hex, $400 \mathrm{MHz}$. Figure S5: C13 NMR spectrum of WA-hex, $100 \mathrm{MHz}$. Figure S6: Botanical description of Physcia mediterranea Nimis collected in El Kala region (Algeria).

Author Contributions: Conceptualisation, M.K. and M.A.A.; methodology of chemical analysis, J.M.L.R., N.S. and M.K.; validation, J.M.L.R., N.S. and M.A.A.; investigation, M.K. and N.S.; writingoriginal draft preparation, R.A.-L., M.K. and M.A.A.; references, R.A.-L., M.K. and M.A.A.; writingreview and editing, R.A.-L.; supervision, J.M.L.R. and M.A.A.; project administration, L.S., J.F.B., C.R., J.M.L.R., and M.A.A.; funding acquisition, J.M.L.R. All authors have read and agreed to the published version of the manuscript.

Funding: The authors thank FibEnTech-UBI, which is financed by National Funds from Fundação para a Ciência e a Tecnologia (FCT) and Community Funds (UID/Multi/00195).

Institutional Review Board Statement: Not applicable.

Informed Consent Statement: Not applicable.

Data Availability Statement: Lichen of Physcia mediterranea Nimis was used in this study. Professor Monia Ali Ahmed identified Physcia mediterranea Nimis, lichenologist and research director of the 
Pathology of Ecosystems team at the University of Badji-Mokhtar, Annaba, Algeria. The identification was confirmed by Pr Jean Michel Sussey, lichenologist at the French Association of Lichenology (AFL). This sample has been deposited in Badji-Mokhtar University, Annaba, code AAM-1.

Acknowledgments: We thank the Ministry of Higher Education and Scientific Research (MESRS) of Algeria for the financial support to this study, and we would like to warmly thank Grira Abdeslem, Principal Forestry Inspector, El Kala National Park, for his presence and his invaluable assistance in the field.

Conflicts of Interest: The authors declare no conflict of interest.

Sample Availability: Samples of all compounds used in the study are available from the authors.

\section{References}

1. Boullard, B. Guerre et Paix Dans le Règne Végétal; Ellipses: Paris, France, 1990.

2. Armaleo, D.; Müller, O.; Lutzoni, F.; Andrésson, Ó.S.; Blanc, G.; Bode, H.B.; Collart, F.R.; Dal Grande, F.; Dietrich, F.; Grigoriev, I.V.; et al. The lichen symbiosis re-viewed through the genomes of Cladonia grayi and its algal partner Asterochloris glomerata. BMC Genom. 2019, 20, 1-33. [CrossRef]

3. Weber, B.; Büdel, B. Fungi and lichens. In Encyclopedia of Earth Sciences Series; Springer: Amsterdam, The Netherlands, 2011; pp. 401-409.

4. Taylor, T.N.; Krings, M. Fossil Plants: Fungi and Lichens. In Encyclopedia of Geology; Elsevier: Amsterdam, The Netherlands, 2004; pp. 436-443. ISBN 9780123693969.

5. Adams, D.G.; Bergman, B.; Nierzwicki-Bauer, S.A.; Duggan, P.S.; Rai, A.N.; Schüßler, A. Cyanobacterial-plant symbioses. In The Prokaryotes: Prokaryotic Biology and Symbiotic Associations; Springer: Berlin/Heidelberg, Germany, 2013 ; pp. 359-400. ISBN 9783642301940.

6. Werth, S.; Sork, V.L. Identity and genetic structure of the photobiont of the epiphytic lichen Ramalina menziesii on three oak species in southern California. Am. J. Bot. 2010, 97, 821-830. [CrossRef] [PubMed]

7. Van-Haluwyn, C.; Asta, J.; Gaveriaux, J.P. Guide des Lichens de France: Lichens Des Arbres; Belin, Ed.; Belin: Paris, France, 2009; ISBN 978-2701147000.

8. Meeßen, J.; Sánchez, F.J.; Brandt, A.; Balzer, E.M.; de la Torre, R.; Sancho, L.G.; de Vera, J.P.; Ott, S. Extremotolerance and Resistance of Lichens: Comparative Studies on Five Species Used in Astrobiological Research I. Morphological and Anatomical Characteristics. Orig. Life Evol. Biosph. 2013, 43, 283-303. [CrossRef] [PubMed]

9. Kranner, I.; Cram, W.J.; Zorn, M.; Wornik, S.; Yoshimura, I.; Stabentheiner, E.; Pfeifhofer, H.W. Antioxidants and photoprotection in a lichen as compared with its isolated symbiotic partners. Proc. Natl. Acad. Sci. USA 2005, 102, 3141-3146. [CrossRef] [PubMed]

10. Reyes, A.; Lopez-Bilbao, M.G.; Del, M.; Molina, C. Relationship between Isozymic Variability and Envir-onmental Conditions in the Lichen Xanthoria parietina. Phyton 1996, 36, 265-275.

11. Goga, M.; Elečko, J.; Marcinčinová, M.; Ručová, D.; Bačkorová, M.; Bačkor, M. Lichen Metabolites: An Overview of Some Secondary Metabolites and Their Biological Potential. In Co-Evolution of Secondary Metabolites; Springer: Cham, Germany, 2018; pp. 1-36.

12. Kaushik, A.; Giri, P.P. Lichens. In Lichen-Derived Products; Wiley: Hoboken, NJ, USA, 2020; pp. 179-187.

13. Upreti, D.K.; Divakar, P.K.; Shukla, V.; Bajpai, R. Recent Advances in Lichenology: Modern Methods and Approaches in Lichen Systematics and Culture Techniques; Springer: New Delhi, India, 2015; Volume 2, ISBN 9788132222354.

14. Yamamoto, Y.; Kinoshita, Y.; Kinoshita, K.; Koyama, K.; Takahashi, K. A zearalenone derivative from the liquid culture of the lichen, Baeomyces placophyllus. J. Hattori Bot. Lab. 2002, 92, 285-289.

15. Chicita, F.C. Chemical and Botanical Guide to Lichen Products; UNC Press: Chapel Hill, NC, USA, 1969; ISBN 978-0-8078-6859-1.

16. Moreira, A.S.N.; Braz-Filho, R.; Mussi-Dias, V.; Vieira, I.J.C. Chemistry and biological activity of Ramalina lichenised fungi. Molecules 2015, 20, 8952-8987. [CrossRef] [PubMed]

17. Oksanen, I. Ecological and biotechnological aspects of lichens. Appl. Microbiol. Biotechnol. 2006, 73, 723-734. [CrossRef]

18. Huneck, S.; Yoshimura, I.; Huneck, S.; Yoshimura, I. Identification of Lichen Substances. In Identification of Lichen Substances; Springer: Berlin/Heidelberg, Germany, 1996; pp. 11-123.

19. Calcott, M.J.; Ackerley, D.F.; Knight, A.; Keyzers, R.A.; Owen, J.G. Secondary metabolism in the lichen symbiosis. Chem. Soc. Rev. 2018, 47, 1730. [CrossRef]

20. Elshobary, M.E.; Osman, M.E.; Abo-Shady, A.M.; Komatsu, E.; Perreault, H.; Sorensen, J.; Piercey-Normore, M.D. Algal carbohydrates affect polyketide synthesis of the lichen-forming fungus Cladonia rangiferina. Mycologia 2016, 108, 646-656. [CrossRef]

21. Elkhateeb, W.; Daba, G. Occurrence of terpenes, polyketides, and tannins in some Japanese lichens and green mosses. Egypt. Pharm. J. 2020, 19, 216. [CrossRef]

22. Solárová, Z.; Liskova, A.; Samec, M.; Kubatka, P.; Büsselberg, D.; Solár, P. Anticancer potential of lichens' secondary metabolites. Biomolecules 2020, 10, 87. [CrossRef] 
23. Jha, B.N.; Shrestha, M.; Pandey, D.P.; Bhattarai, T.; Bhattarai, H.D.; Paudel, B. Investigation of antioxidant, antimicrobial and toxicity activities of lichens from high altitude regions of Nepal. BMC Complement. Altern. Med. 2017, 17, 1-8. [CrossRef] [PubMed]

24. Thadhani, V.M.; Karunaratne, V. Potential of Lichen Compounds as Antidiabetic Agents with Antioxidative Properties: A Review. Oxid. Med. Cell. Longev. 2017, 2017. [CrossRef] [PubMed]

25. Rodriguez, C.M.; Bennett, J.P.; Johnson, C.J. Lichens: Unexpected anti-prion agents? Prion 2012, 6, 11-16. [CrossRef]

26. Millot, M.; Girardot, M.; Dutreix, L.; Mambu, L.; Imbert, C. Antifungal and anti-biofilm activities of acetone lichen extracts against Candida albicans. Molecules 2017, 22, 651. [CrossRef] [PubMed]

27. Zambare, V.P.; Christopher, L.P. Biopharmaceutical potential of lichens. Pharm. Biol. 2012, 50, 778-798. [CrossRef] [PubMed]

28. White, P.A.S.; Oliveira, R.C.M.; Oliveira, A.P.; Serafini, M.R.; Araújo, A.A.S.; Gelain, D.P.; Moreira, J.C.F.; Almeida, J.R.G.S.; Quintans, J.S.S.; Quintans-Junior, L.J.; et al. Antioxidant activity and mechanisms of action of natural compounds isolated from lichens: A systematic review. Molecules 2014, 19, 14496-14527. [CrossRef]

29. Shukla, V.; Joshi, G.P.; Rawat, M.S.M. Lichens as a potential natural source of bioactive compounds: A review. Phytochem. Rev. 2010, 9, 303-314. [CrossRef]

30. Behera, B.C.; Mahadik, N.; Morey, M. Antioxidative and cardiovascular-protective activities of metabolite usnic acid and psoromic acid produced by lichen species Usnea complanata under submerged fermentation. Pharm. Biol. 2012, 50, 968-979. [CrossRef]

31. Crawford, S.D. Lichens used in traditional medicine. In Lichen Secondary Metabolites: Bioactive Properties and Pharmaceutical Potential; Springer International Publishing: Berlin/Heidelberg, Germany, 2015; pp. 27-80. ISBN 9783319133744.

32. Crawford, S.D. Lichens Used in Traditional Medicine. In Lichen Secondary Metabolites; Springer International Publishing: Berlin/Heidelberg, Germany, 2019; pp. 31-97.

33. Komaty, S.; Letertre, M.; Dang, D.; Jungnickel, H.; Laux, P. Sample preparation for an optimised extraction of localised metabolites in lichens: Application to Pseudevernia furfuracea. Talanta 2015. [CrossRef]

34. Rafika, B.; Monia, A. Antibacterial Activity of the Chloroform, Acetone, Methanol and queous Extracts of Algerian Lichens. Jordan J. Pharm. Sci. 2018, 11, 55-67.

35. Monia, A.A.; Rafika, B.; Tarek, H. Lichen diversity in the edough peninsula, North East of Algeria. Bot. Complut. 2018, 42, 9-18. [CrossRef]

36. Brakni, R.; Serradj, M.; Fernandez, X.; Michel, T. Chemical and biological investigation of Algerian lichens. Planta Med. 2016, 82, P657. [CrossRef]

37. Aljabeili, H.S.; Barakat, H.; Abdel-Rahman, H.A. Chemical Composition, Antibacterial and Antioxidant Activities of Thyme Essential Oil (Thymus vulgaris). Food Nutr. Sci. 2018, 9, 433-446. [CrossRef]

38. Serradj, M.; El Oualidi, J.; Slimani, A.; Boumedris, Z. Contribution to the lichens;inventory from the Oubeira Lake (NE Algeria). Bull. Inst. Sci. Rabat Sect. Sci. Vie 2013, 35, 15-17.

39. Amrani, S.; Nacer, A.; Noureddine, N.E.; Seaward, M.R. Lichenological exploration of Algeria: Historical overview and annotated bibliography, 1799-2013. Willdenowia 2015, 45, 15-34. [CrossRef]

40. Rebbas, K.; Boutabia, L.; Touazi, Y.; Gharzouli, R.; Djellouli, Y.; Alatou, D. Inventaire des lichens du Parc national de Gouraya (Béjaïa, Algérie). Phytotherapie 2011, 9, 225-233. [CrossRef]

41. Mohamed, A.H.; Miara, M.D.; Rebbas, K.; Slimani, A.; Ravera, S.; Hamerelain, A.S. Mise à jour de l'inventaire des lichens d'Algérie. Rev. Ecol. 2014, 10, 75-103.

42. Rahali, M. Cartographie de la pollution plombique dans la région d'Alger en utilisant un lichen (Xanthoria parientina) comme bioaccumulateur. Pollut. Atmos. 2002, 421-432. [CrossRef]

43. Khedim, R.; Maatoug, M.; Benhassaini, H.; Hammou, M.A. Macrolichens New to Algeria and Other Interesting Species from Theniet-el-Had National Park. Herzogia 2018, 31, 252-267. [CrossRef]

44. Brakni, R.; Ali Ahmed, M.; Burger, P.; Schwing, A.; Michel, G.; Pomares, C.; Hasseine, L.; Boyer, L.; Fernandez, X.; Landreau, A.; et al. UHPLC-HRMS/MS Based Profiling of Algerian Lichens and Their Antimicrobial Activities. Chem. Biodivers. 2018, 15, e1800031. [CrossRef] [PubMed]

45. Slimani, A.; Ahmed, A.; Serradj, M.; Hamel, T.; Coste, C. Contribution a l'etude de la flore lichenique dans la zeenaie de Bougous (foret de Ramel Tonal) au niveau du Pare National d'El Kala Nord Est Algerien. Synthèse Rev. Des Sci. Et De La Technol. 2013, 27, 22-29.

46. Bouiadjra, S.B.; Ramdani, M.; Djellouli, R.; ELkhiati, N.; Zerey, W.E.; Bouterfas, K.; Roger, F. Air quality in the metropolitan area of Sidi Bel Abbes (Algeria) through the lichens species as bio-indicators. Moroc. J. Chem. 2017, 5. [CrossRef]

47. Ghennam, K.; Fatiha, A. Biosurveillance par SIG de la qualité de l'air d'une cité méditerraneene (Alger), diversité lichénique, indice de pureté atmosphérique (IPA) et indice d'impact. Revue d'Écologie 2017, 72, 134-146.

48. Maizi, N.; Alioua, A.; Tahar, A. Jumelage des bio-indicateurs et d'un réseau de surveillance de la qualité de l'air pour la détection de la pollution par le SO 2 dans la région de Annaba (Algérie). Biotechnol. Agron. Soc. Env. 2012, 16, 149-158.

49. Serradj-Ali Ahmed, M.; Boumedris, Z.E.; Djebar, M.R.; Tahar, A. Responses of antioxidants in Flavoparmelia caperata (L.) Hale to the atmospheric pollution air at two urban and semi-urban areas in the region of Annaba (East of Algeria). Pollut. Atmos. 2014. [CrossRef]

50. Djamel, S.; Yamna, D.; Djamel, A. Biological diversity of the National Park of El-Kala (Algeria), valorisation and protection. Biodivers. J. 2014, 5, 525-532. 
51. Putra, P.P.; Abdullah, S.S.; Rahmatunisa, R.; Junaidin, J.; Ismed, F. Structure, activity, and drug-likeness of pure compounds of Sumatran lichen (Stereocaulon halei) for the targeted ACE2 protein in COVID-19 disease. Pharmaciana 2020, 10, 135. [CrossRef]

52. Mallavadhani, U.V.; Tirupathamma, R.S.; Sagarika, G.; Ramakrishna, S. Isolation, Chemical Modification, and Anticancer Activity of Major Metabolites of the Lichen Parmotrema mesotropum. Chem. Nat. Compd. 2019, 55, 825-831. [CrossRef]

53. Nishiyama, Y.; Noda, Y.; Nakatani, N.; Shitan, N.; Sudo, T.; Kato, A.; Mutiso, P.B.C. Structure of constituents isolated from the bark of Cassipourea malosana and their cytotoxicity against a human ovarian cell line. J. Nat. Med. 2019, 73, 289-296. [CrossRef] [PubMed]

54. Vladimirova, I.N.; Georgiyants, V.A. Extracted compounds from Cetraria islandica. Chem. Nat. Compd. 2013, 49, 347-348. [CrossRef]

55. Akbar, S.; Akbar, S. Dolichousnea longissima (Ach) Articus/Parmelia perlata Esch. (Parmeliaceae). In Handbook of 200 Medicinal Plants; Springer International Publishing: Berlin/Heidelberg, Germany, 2020; pp. 873-878.

56. Yu, X.L.; Yang, X.Y.; Gao, X.L.; Bai, R.F.; Yin, X.; Su, G.Z.; Qu, C.H.; Chai, X.Y.; Tu, P.F. Phenolic constituents from lichen Usnea longissima. Zhongguo Zhongyao Zazhi 2016, 41, 1864-1869. [CrossRef] [PubMed]

57. Carpentier, C.; Queiroz, E.F.; Marcourt, L.; Wolfender, J.L.; Azelmat, J.; Grenier, D.; Boudreau, S.; Voyer, N. Dibenzofurans and pseudodepsidones from the lichen Stereocaulon paschale collected in northern Quebec. J. Nat. Prod. 2017, 80, 210-214. [CrossRef]

58. Ari, F.; Ulukaya, E.; Oran, S.; Celikler, S.; Ozturk, S.; Ozel, M.Z. Promising anticancer activity of a lichen, Parmelia sulcata Taylor, against breast cancer cell lines and genotoxic effect on human lymphocytes. Cytotechnology 2015, 67, 531-543. [CrossRef] [PubMed]

59. Stojanović, I.Ž.; Radulović, N.S.; Mitrović, T.L.; Stamenković, S.M.; Stojanović, G.S. Volatile constituents of selected Parmeliaceae lichens. J. Serb. Chem. Soc. 2011, 76, 987-994. [CrossRef]

60. Hoffmann, H.R.; Matusch, R.; Baniahmad, A. Isolation of Atraric Acid, Synthesis of Atraric Acid Derivatives, and Use of Atraric Acid and the Derivatives thereofe for the Treatment of Benigne Prostatic Hyperplasia, Prostate Carcinoma and Spinobulbar Muscular Atrophy. U.S. Patent 8,481,519, 9 July 2013.

61. Hessenkemper, W.; Roediger, J.; Bartsch, S.; Houtsmuller, A.B.; van Royen, M.E.; Petersen, I.; Grimm, M.-O.; Baniahmad, A. A natural androgen receptor antagonist induces cellular senescence in prostate cancer cells. Mol. Endocrinol. 2014, 28, 1831-1840. [CrossRef]

62. Güvenç, A.; Akkol, E.K.; Süntar, I.; Keleş, H.; Yildiz, S.; Çaliş, I. Biological activities of Pseudevernia furfuracea (L.) Zopf extracts and isolation of the active compounds. J. Ethnopharmacol. 2012, 144, 726-734. [CrossRef]

63. Ahad, A.M.; Goto, Y.; Kiuchi, F.; Tsuda, Y.; Kondo, K.; Sato, T. Nematocidal principles in "Oakmoss Absolute" and nematocidal activity of 2, 4-Dihydroxybenzoates. Chem. Pharm. Bull. 1991, 39, 1043-1046. [CrossRef]

64. Yamamoto, Y.; Watanabe, A. Fatty acid their composition of lichens and their phyco-and mycobionts. J. Gen. Appl. Microbiol. 1974, 20, 83-86. [CrossRef]

65. Sonesson, M.; Grimberg, Å.; Sveinbjörnsson, B.; Carlsson, B.Å. Seasonal variation in concentrations of carbohydrates and lipids in two epiphytic lichens with contrasting, snow-depth related distribution on subarctic birch trees. Bryologist 2011, 114, 443-452 [CrossRef]

66. ̌ezanka, T.; Dembitsky, V.M. Fatty acids of lichen species from Tian Shan Mountains. Folia Microbiol. 1999, 44, 643-646. [CrossRef]

67. Vu, T.H.; Catheline, D.; Delmail, D.; Boustie, J.; Legrand, P.; Lohezic-Le Devehat, F. Gas chromatographic analysis to compare the fatty acid composition of fifteen lichen species, with a focus on Stereocaulon. Lichenologist 2016, 48, 323-337. [CrossRef]

68. Culberson, C. Biogenetic relationships of the lichen substances in the framework of systematics. Bryologist 1986, 89, 91-98. [CrossRef]

69. Fehrer, J.; Slavíková-Bayerová, Š.; Orange, A. Large genetic divergence of new, morphologically similar species of sterile lichens from Europe (Lepraria, Stereocaulaceae, Ascomycota): Concordance of DNA sequence data with secondary metabolites. Cladistics 2008, 24, 443-458. [CrossRef]

70. Hale, M.E. A Monograph of the Lichen Genus Parmelia Acharius sensu Strict (Ascomycotina: Pameliaceae); Smithsonian Institution Press: Washington, DC, USA, 1987.

71. Haugan, R.; Timdal, E. Tephromela perlata and T. talayana, with notes on the T. aglaea-complex. Graph. Scr. 1994, 6, 17-26.

72. Spribille, T.; Klug, B.; Mayrhofer, H. A phylogenetic analysis of the boreal lichen Mycoblastus sanguinarius (Mycoblastaceae, lichenised Ascomycota) reveals cryptic clades correlated with fatty acid profiles. Mol. Phylogenet. Evol. 2011, 59, 603-614. [CrossRef] [PubMed]

73. Lumbsch, H.T. The Use of Metabolic Data in Lichenology at the Species and Subspecific Levels. Lichenologist 1998, 30, 357-367. [CrossRef]

74. Hawksworth, D.L. Lichen chemotaxonomy. In Lichenology: Progress and Problems; Proceedings of an international Symposium; Academic Press: Cambridge, MA, USA, 1976; pp. 139-184.

75. Stocker-Wörgötter, E. Metabolic diversity of lichen-forming ascomycetous fungi: Culturing, polyketide and shikimate metabolite production, and PKS genes. Nat. Prod. Rep. 2008, 25, 188-200. [CrossRef]

76. Sumida, C.; Graber, R.; Nunez, E. Role of fatty acids in signal transduction: Modulators and messengers. Prostaglandins Leukot. Essent. Fat. Acids 1993, 48, 117-122. [CrossRef]

77. Bychek-Gushchina, I. Study of biochemical aspects of lichen symbiosis. I. Lipids and fatty acids in cultured lichen symbionts. Biochemistry 1997, 62, 490-494. 
78. Torres, A.; Dor, I.; Rotem, J.; Srebnik, M.; Dembitsky, V.M. Characterization of surface n-alkanes and fatty acids of the epiphytic lichen Xanthoria parietina, its photobiont a green alga Trebouxia sp., and its mycobiont, from the Jerusalem hills. Eur. J. Biochem. 2003, 270, 2120-2125. [CrossRef] [PubMed]

79. Molina, M.C.; Crespo, A.; Vicente, C.; Elix, J.A. Differences in the composition of phenolics and fatty acids of cultured mycobiont and thallus of Physconia distorta. Plant Physiol. Biochem. 2003, 41, 175-180. [CrossRef]

80. Dembitsky, V.M.; Rezanka, T.; Bychek, I.A. Seasonal variation of lipids and fatty acids from tree-growing lichens of the genus Physcia. Phytochemistry 1994, 36, 601-608. [CrossRef]

81. Dembitsky, V.M.; Rezanka, T.; Bychek, I. A Seasonal variability of lipids and fatty acids in the tree-growing lichen Xanthoria parientina L. J. Exp. Bot. 1994, 45, 403-408. [CrossRef]

82. Reis, R.A.; Iacomini, M.; Gorin, P.A.J.; Souza, L.M.; Grube, M.; Cortes Cordeiro, L.M.; Sassaki, G.L. Fatty acid composition of the tropical lichen Teloschistes flavicans and its cultivated symbionts. FEMS Microbiol. Lett. 2005, 247, 1-6. [CrossRef]

83. Goss, R.; Wilhelm, C. Lipids in Algae, Lichens and Mosses. In Lipids in Photosynthesis; Springer: Dordrecht, The Netherlands, 2009; pp. 117-137.

84. Temina, M.; Levitsky, D.O.; Dembitsky, V.M. Chemical Constituents of the Epiphytic and Lithophilic Lichens of the Genus Collema. Rec. Nat. Prod. 2010, 4, 79-86.

85. Ilica, R.A.; Kloetzer, L.; Galaction, A.I.; Caşcaval, D. Fumaric acid: Production and separation. Biotechnol. Lett. 2019, 41, 47-57. [CrossRef] [PubMed]

86. Sebastian, J.; Hegde, K.; Kumar, P.; Rouissi, T.; Brar, S.K. Bioproduction of fumaric acid: An insight into microbial strain improvement strategies. Crit. Rev. Biotechnol. 2019, 39, 817-834. [CrossRef]

87. Haghikia, A.; Linker, R.; Gold, R. Fumarsäure in der Therapie der Multiplen Sklerose. Nervenarzt 2014, 85, 720-726. [CrossRef] [PubMed]

88. Papadaki, A.; Papapostolou, H.; Alexandri, M.; Kopsahelis, N.; Papanikolaou, S.; de Castro, A.M.; Freire, D.M.G.; Koutinas, A.A. Fumaric acid production using renewable resources from biodiesel and cane sugar production processes. Environ. Sci. Pollut. Res. 2018, 25, 35960-35970. [CrossRef] [PubMed]

89. Jabłońska-Trypuć, A.; Pankiewicz, W.; Czerpak, R. Traumatic Acid Reduces Oxidative Stress and Enhances Collagen Biosynthesis in Cultured Human Skin Fibroblasts. Lipids 2016, 51, 1021-1035. [CrossRef]

90. Jabłońska-Trypuć, A.; Krętowski, R.; Wołejko, E.; Wydro, U.; Butarewicz, A. Traumatic acid toxicity mechanisms in human breast cancer MCF-7cells. Regul. Toxicol. Pharmacol. 2019, 106, 137-146. [CrossRef] [PubMed]

91. Lee, H.C.; Liu, F.C.; Tsai, C.N.; Chou, A.H.; Liao, C.C.; Yu, H.P. Esculetin Ameliorates Lipopolysaccharide-Induced Acute Lung Injury in Mice Via Modulation of the AKT/ERK/NF-kB and ROR $\gamma \mathrm{t} / \mathrm{IL}-17$ Pathways. Inflammation 2020, 43, 962-974. [CrossRef] [PubMed]

92. Wang, C.; Pei, A.; Chen, J.; Yu, H.; Sun, M.L.; Liu, C.F.; Xu, X. A natural coumarin derivative esculetin offers neuroprotection on cerebral ischemia/reperfusion injury in mice. J. Neurochem. 2012, 121, 1007-1013. [CrossRef]

93. Hongyan, L. Esculetin Attenuates Th2 and Th17 Responses in an Ovalbumin-Induced Asthmatic Mouse Model. Inflammation 2016, 39, 735-743. [CrossRef] [PubMed]

94. Han, M.H.; Park, C.; Lee, D.S.; Hong, S.H.; Choi, I.W.; Kim, G.Y.; Choi, S.H.; Shim, J.H.; Chae, J.I.; Yoo, Y.H.; et al. Cytoprotective effects of esculetin against oxidative stress are associated with the upregulation of Nrf2-mediated NQO1 expression via the activation of the ERK pathway. Int. J. Mol. Med. 2017, 39, 380-386. [CrossRef]

95. Srikrishna, D.; Godugu, C.; Dubey, P.K. A Review on Pharmacological Properties of Coumarins. Mini-Rev. Med. Chem. 2016, 18, 113-141. [CrossRef] [PubMed]

96. Stefanachi, A.; Leonetti, F.; Pisani, L.; Catto, M.; Carotti, A. Coumarin: A Natural, Privileged and Versatile Scaffold for Bioactive Compounds. Molecules 2018, 23, 250. [CrossRef]

97. Musa, M.; Cooperwood, J.; Khan, M.O. A Review of Coumarin Derivatives in Pharmacotherapy of Breast Cancer. Curr. Med. Chem. 2008, 15, 2664-2679. [CrossRef]

98. Studzińska-Sroka, E.; Galanty, A.; Bylka, W. Atranorin-an interesting lichen secondary metabolite. Mini-Rev. Med. Chem. 2017, 17, 1633-1645. [CrossRef] [PubMed]

99. Huneck, S.; Feige, G.B.; Schmidt, J. Chemie von Cladonia furcata und Cladonia rangiformis. Herzogia 2004, 17, 51-58.

100. Nybakken, L.; Helmersen, A.M.; Gauslaa, Y.; Selås, V. Lichen compounds Restrain lichen feeding by bank voles (Myodes glareolus). J. Chem. Ecol. 2010, 36, 298-304. [CrossRef]

101. Culberson, C.F. Chemical and Botanical Guide to Lichen Products, 2nd ed.; Otto Koeltz Science Publishers: Koenigstein, Germany, 1979.

102. Elix, J.A. A Catalogue of Standardized Chromatographic Data and Biosynthetic Relationships for Lichen Substances; Australian National University: Canberra, Australia, 2014.

103. Ranković, B.; Mišić, M.; Sukdolak, S. The antimicrobial activity of substances derived from the lichens Physcia aipolia, Umbilicaria polyphylla, Parmelia caperata and Hypogymnia physodes. World J. Microbiol. Biotechnol. 2008, 24, 1239-1242. [CrossRef]

104. Solhaug, K.A.; Larsson, P.; Gauslaa, Y. Light screening in lichen cortices can be quantified by chlorophyll fluorescence techniques for both reflecting and absorbing pigments. Planta 2010, 231, 1003-1011. [CrossRef]

105. Brodo, I.M.; Freebury, C.; Alfonso, N. Notes on the Lichens Physcia aipolia and P. alnophila in North America. Evansia 2013, 30 , 110-119. [CrossRef] 
106. Maldonado Montaño, A.; Menesses, R.; Bravo, J.A.; Vila, J.L.; Bello, A. Presence of atranorin in Physcia sorediosa. Rev. Boliv. Quím. 2016, 33, 175-178.

107. Kosanić, M.; Ranković, B.; Stanojković, T.; Rančić, A.; Manojlović, N. Cladonia lichens and their major metabolites as possible natural antioxidant, antimicrobial and anticancer agents. LWT Food Sci. Technol. 2014, 59, 518-525. [CrossRef]

108. Pompilio, A.; Pomponio, S.; Di Vincenzo, V.; Crocetta, V.; Nicoletti, M.; Piovano, M.; Garbarino, J.A.; Di Bonaventura, G. Antimicrobial and antibiofilm activity of secondary metabolites of lichens against methicillin-resistant Staphylococcus aureus strains from cystic fibrosis patients. Future Microbiol. 2013, 8, 281-292. [CrossRef] [PubMed]

109. Melo, M.G.D.; Araújo, A.A.S.; Serafini, M.R.; Carvalho, L.F.; Bezerra, M.S.; Ramos, C.S.; Bonjardim, L.R.; Albuquerque-Júnior, R.L.C.; Lima, J.T.; Siqueira, R.S.; et al. Anti-inflammatory and toxicity studies of atranorin extracted from Cladina kalbii Ahti in rodents. Braz. J. Pharm. Sci. 2011, 47, 861-872. [CrossRef]

110. Melo, M.G.D.; Araújo, A.A.S.; Rocha, C.P.L.; Almeida, E.M.S.A.; De Souza Siqueira, R.; Bonjardim, L.R.; Quintans-Júnior, L.J. Purification, physicochemical properties, thermal analysis and antinociceptive effect of atranorin extracted from Cladina kalbii. Biol. Pharm. Bull. 2008, 31, 1977-1980. [CrossRef]

111. Barreto, R.S.S.; Albuquerque-Júnior, R.L.C.; Pereira-Filho, R.N.; Quintans, J.S.S.; Barreto, A.S.; DeSantana, J.M.; Santana-Filho, V.J.; Santos, M.R.V.; Bonjardim, L.R.; Araújo, A.A.S.; et al. Evaluation of wound healing activity of atranorin, a lichen secondary metabolite, on rodents. Braz. J. Pharmacogn. 2013, 23, 310-319. [CrossRef]

112. Harikrishnan, A.; Veena, V.; Lakshmi, B.; Shanmugavalli, R.; Theres, S.; Prashantha, C.N.; Shah, T.; Oshin, K.; Togam, R.; Nandi, S. Atranorin, an antimicrobial metabolite from lichen Parmotrema rampoddense exhibited in vitro anti-breast cancer activity through interaction with Akt activity. J. Biomol. Struct. Dyn. 2020. [CrossRef] [PubMed]

113. Galanty, A.; Koczurkiewicz, P.; Wnuk, D.; Paw, M.; Karnas, E.; Podolak, I.; Węgrzyn, M.; Borusiewicz, M.; Madeja, Z.; Czyż, J.; et al. Usnic acid and atranorin exert selective cytostatic and anti-invasive effects on human prostate and melanoma cancer cells. Toxicol. Vitr. 2017, 40, 161-169. [CrossRef] [PubMed]

114. Hikino, H.; Ito, K.; Takemoto, T. Stereostructure of Asebotoxin I and II, Toxins of Pieris japonica. Chem. Pharm. Bull. 1969, 17, 854-855. [CrossRef] [PubMed]

115. Zhang, L.; Hong, Z.; Zhang, R.R.; Sun, X.Z.; Yuan, Y.F.; Hu, J.; Wang, X. Bakkenolide A inhibits leukemia by regulation of HDAC3 and PI3K/Akt-related signaling pathways. Biomed. Pharmacother. 2016, 83, 958-966. [CrossRef]

116. Solberg, Y. Chemical constituents of the lichen species Cetraria islandica. J. Hattori Bot. Lab. 1986, 60, 391-406.

117. Simirgiotis, M.J.; Quispe, C.; Areche, C.; Sepúlveda, B. Phenolic compounds in Chilean Mistletoe (quintral, Tristerix tetrandus) analysed by UHPLC-Q/Orbitrap/MS/MS and its antioxidant properties. Molecules 2016, 21, 245. [CrossRef]

118. Huneck, S. New Results on the Chemistry of Lichen Substances. In Progress in the Chemistry of Organic Natural Products; Springer: Berlin/Heidelberg, Germany, 2001; Volume 81.

119. Seo, D.Y.; Lee, S.R.; Heo, J.W.; No, M.H.; Rhee, B.D.; Ko, K.S.; Kwak, H.B.; Han, J. Ursolic acid in health and disease. Korean J. Physiol. Pharmacol. 2018, 22, 235-248. [CrossRef]

120. Gonzalez, A.G.; Bermejo Barrera, J.; Rodriguez Perez, E.M.; Hernandez Padron, C.E. chemical constituents of the lichen Ramalina hierrensis. Planta Med. 1992, 58, 214-218. [CrossRef] [PubMed]

121. Vu, T.H.; Lamer, A.C.L.; Lalli, C.; Samson, J.B.M.; Dévéhat, F.L.L.; Seyec, J. Le Depsides: Lichen metabolites active against hepatitis C virus. PLoS ONE 2015, 10, e0120405. [CrossRef] [PubMed]

122. Rubio, C.; Galloway, D.J.; Quilhot, W. Secondary products from Pannaria tavaresii (Lichens). J. Chil. Chem. Soc. 2005, 50, 667-669. [CrossRef]

123. De Carvalho, M.G.; De Carvalho, G.J.A.; Braz-Filho, R. Chemical Constituents from Ouratea floribunda: Complete 1H and 13C NMR Assignments of Atranorin and its New Acetyl Derivative. J. Braz. Chem. Soc. 2000, 11, 143-147. [CrossRef]

124. Norouzi, H.; Azizi, A.; Gholami, M.; Sohrabi, M.; Boustie, J. Chemotype variations among lichen ecotypes of Umbilicaria aprina as revealed by LC-ESI-MS/MS: A survey of antioxidant phenolics. Environ. Sci. Pollut. Res. 2020, 27, 40296-40308. [CrossRef] [PubMed]

125. Vos, C.; McKinney, P.; Pearson, C.; Heiny, E.; Gunawardena, G.; Holt, E.A. The optimal extraction and stability of atranorin from lichens, in relation to solvent and $\mathrm{pH}$. Lichenologist 2018, 50, 499-512. [CrossRef]

126. Lohezic-Le Devehat, F.; Legouin, B.; Couteau, C.; Boustie, J.; Coiffard, L. Lichenic extracts and metabolites as UV filters. J. Photochem. Photobiol. B Biol. 2013, 120, 17-28. [CrossRef]

127. Bjerke, J.W.; Elvebakk, A.; Domínguez, E.; Dahlback, A. Seasonal trends in usnic acid concentrations of Arctic, alpine and Patagonian populations of the lichen Flavocetraria nivalis. Phytochemistry 2005, 66, 337-344. [CrossRef]

128. Marante, F.J.T.; Castellano, A.G.; Rosas, F.E.; Aguiar, J.Q.; Barrera, J.B. Identification and quantitation of allelochemicals from the lichen Lethariella canariensis: Phytotoxicity and antioxidative activity. J. Chem. Ecol. 2003, 29, 2049-2071. [CrossRef]

129. Solhaug, K.A.; Lind, M.; Nybakken, L.; Gauslaa, Y. Possible functional roles of cortical depsides and medullary depsidones in the foliose lichen Hypogymnia physodes. Flora Morphol. Distrib. Funct. Ecol. Plants 2009, 204, 40-48. [CrossRef]

130. Forbes, P.; Wat, L.; Strumpher, J. Comparative Perspectives on Extraction Methods for Organic Metabolites and Pollutants from Lichens. In Lichen-Derived Products; Wiley: Hoboken, NJ, USA, 2020; pp. $27-73$.

131. Augusto, S.; Pinho, P.; Branquinho, C.; Pereira, M.J.; Soares, A.; Catarino, F. Atmospheric Dioxin and Furan Deposition in Relation to Land-Use and Other Pollutants: A Survey with Lichens; Kluwer Academic Publishers: Dordrecht, The Netherlands, 2004; Volume 49. 
132. Van der Wat, L.; Forbes, P.B.C. Comparison of extraction techniques for polycyclic aromatic hydrocarbons from lichen biomonitors. Environ. Sci. Pollut. Res. 2019, 26, 11179-11190. [CrossRef]

133. Van Der Wat, L.; Forbes, P.B.C. Lichens as biomonitors for organic air pollutants. TrAC Trends Anal. Chem. 2015, 64, 165-172. [CrossRef]

134. Caccamese, S.; Toscano, R.M.; Bellesia, F.; Pinetti, A. Methyl $\beta$-Orcinolcarboxylate and Depsides from Parmelia Furfuracea. J. Nat. Prod. 1985, 48, 157-158. [CrossRef]

135. Gunzinger, J.; Tabacchi, R. Isolement et identification de l'acide furfurique, nouvelle depsidone du lichen Pseudevernia furfuracea (L.) Ach. Helv. Chim. Acta 1985, 68, 1936-1939. [CrossRef]

136. Culberson, W.L.; Culberson, C.F.; Johnson, A. Pseudevernia furfuracea-Olivetorina Relationships: Chemistry and Ecology. Mycologia 1977, 69, 604. [CrossRef]

137. Joulain, D.; Tabacchi, R. Lichen extracts as raw materials in perfumery. Part 2: Treemoss. Flavour Fragr. J. 2009, $24,105-116$. [CrossRef] 